\title{
The Use of Added Salt and Sugar in the Diet of Polish and Austrian Toddlers. Associated Factors and Dietary Patterns, Feeding and Maternal Practices
}

\author{
Daria Masztalerz-Kozubek ${ }^{1}$, Monika A. Zielinska ${ }^{1}{ }^{1}$, Petra Rust ${ }^{2}{ }^{\oplus}$, Dorota Majchrzak ${ }^{2}$ \\ and Jadwiga Hamulka $1, *$ (D) \\ 1 Department of Human Nutrition, Institute of Human Nutrition Sciences, Warsaw University of Life \\ Sciences (SGGW-WULS), 02-787 Warsaw, Poland; daria_masztalerz_kozubek@sggw.edu.pl (D.M.-K.); \\ monika_zielinska@sggw.edu.pl (M.A.Z.) \\ 2 Department of Nutritional Sciences, University of Vienna, 1090 Vienna, Austria; \\ petra.rust@univie.ac.at (P.R.); dorota.majchrzak@univie.ac.at (D.M.) \\ * Correspondence: jadwiga_hamulka@sggw.edu.pl; Tel.: +48-22-593-71-12
}

Received: 8 June 2020; Accepted: 9 July 2020; Published: 13 July 2020

\begin{abstract}
Children aged $<2$ years should not be given meals with the addition of salt and sugar due to health risks and to promote healthier dietary habits. The aims of this study were: to assess the prevalence of the use of added salt (AS), sugar (ASu) and both salt and sugar (AS\&Su) in the diets of Polish and Austrian toddlers aged 12-24 and 25-36 months; to explore the sociodemographic and early nutritional factors associated with the use of AS and ASu; to investigate the difference in dietary habits and maternal concerns about toddlers' eating regarding the use of AS and ASu in toddlers' diet. This cross-sectional anonymous study was conducted in 5893 mothers of children aged 12-36 months, recruited through social media in 2017-2019. The questionnaire consisted of questions about sociodemographics, early feeding practices and current children's nutrition (e.g., use of AS and $\mathrm{ASu}$, food frequency questionnaire). Multivariate logistic regression and cluster analyses were applied. Austrian mothers more often used AS than mothers from Poland (at 2 years old: $74.8 \%$ vs. $52.8 \%$; at 3 years old $87.4 \%$ vs. $74.4 \%, p \leq 0.001$ ), however Polish mothers were more prone to use ASu (at 2 years old: $34.7 \%$ vs. $27.7 \%$; at 3 years old: $59.0 \%$ vs. $45.8 \%, p \leq 0.001$ ). In younger toddlers (12-24 months), the odds of using of AS, ASu, and AS\&Su increased with toddlers' age, when the mother was a multipara, was not currently breastfeeding, or had exclusively breastfed for 4-5 months. This risk decreased when older toddlers (25-36 months) were introduced to solids by baby-led weaning (BLW). Toddlers from both countries who consumed meals with AS or ASu more often a followed Western-like dietary pattern. Our study emphasizes the need for parental nutritional education when beginning to introduce solid foods.
\end{abstract}

Keywords: baby-led weaning (BLW); breastfeeding; complementary feeding; complementary foods; dietary patterns; infant feeding practices; maternal concerns; salt; sugar

\section{Introduction}

The first 1000 days of children's intensive growth and development are pivotal times during a life course. According to the Developmental Origins of Health and Disease hypothesis (DOHaD), environmental exposures during prenatal life, infancy and toddlerhood can modify the risk of many metabolic and non-communicable diseases in further life [1]. Moreover, this time is considered a sensitive period for establishing healthy dietary habits [2]. Breastfeeding, complementary feeding, parental feeding practices, but also social and family environment, play a crucial role in the development of children's food preferences, attitudes, and dietary patterns [2-4]. However, children are evolutionarily 
predisposed to prefer high-energy, sweet and salty foods [2,5], as well as to reject bitter or new food items which may be extremely challenging for parents. Additionally, it was reported that repeated exposure to healthy foods (e.g., vegetables) along with limited access to unhealthy ones is a powerful factor that contributes to the development of healthier food preferences and dietary habits that may provide long term health outcomes [2-4]. Nonetheless, it emphasizes the need to offer children foods that are nutrient-dense and appropriate for them $[2,4,6]$.

Health organizations and experts are in agreement that salt and sugar should be avoided during the first two years of life and limited later on [7-10]. Sodium and sugar intake in childhood was linked with many adverse health effects, including high blood pressure [11] and cardiovascular diseases [12], obesity [13,14] or dental caries [15]. Interestingly, in animal studies, it was reported that salt and sugar consumption may alter gut microbiota [16,17], which seems to play a pivotal role in health programming [18]. Another possible implication of early exposure to salt and sugar refers to the development of preference for salty and sweet tastes. It has been suggested that exposure to salty or sweet foods leads to an increase in acceptance and preference of saltiness and sweetness, but results are equivocal $[5,6,19]$. Moreover, adaptation to salty and sweet tastes during childhood may be transferred into adulthood, leading to higher sodium and free sugars intake throughout the lifespan $[5,20]$. Previous studies reported that sodium and added sugar intake increases with age by $\sim 250 \mathrm{mg}$ of salt/day per year from the age of 5 years [21] and from $2.3 \%$ of energy from added sugars in infancy to $13.4 \%$ in primary school pupils [22].

Previous studies that mainly assessed the intake of sodium, free or added sugars and use of added salt and sugar provide explicit results that the majority of young children may have exceeded sodium and free/added sugars recommendations since the earliest periods when they began eating solids [22-28]. As early introduction of those ingredients is a risk factor of many diet-related non-communicable diseases, which are a public health challenge worldwide [29,30], recognition of the factors associated with the use of salt and sugar is highly important. However, there is still little research examining concurrent use of added salt, sugar and both salt and sugar, especially according to various factors, such as sociodemographic or feeding-related. Hence, to fill the aforementioned scientific gaps, the aims of this study were: (1) to assess the prevalence of use of AS, ASu and AS\&Su in the diets of Polish and Austrian toddlers aged 12-24 and 25-36 months; (2) to explore the sociodemographic and early nutritional factors associated with the use of AS, ASu and AS\&Su in toddlers' diets; (3) to investigate the difference in dietary habits and maternal concerns about toddlers' eating regarding the use of AS, ASu and AS\&Su in toddlers' diets. The study was conducted in Poland and Austria, because of differences in complementary feeding practices in both countries as well as sociodemographic differences regarding income, maternity (parental) leave and family structure.

\section{Materials and Methods}

\subsection{Study Design and Participants}

This Internet-based cross-sectional study was conducted between 2017 and 2019 and investigated mothers of toddlers aged 12-36 months from Poland and Austria. The study design, methods and sample selection were described in detail in a previously published paper [31]. The study was performed in compliance with the Helsinki Declaration. As all data were collected anonymously and IP addresses were not collected following Polish and Austrian law, our study did not need ethical approval.

Participants were recruited via social media. They received information about the anonymity of the study, the voluntariness and the possibility to stop their participation at any study stage. Questionnaires in both Polish and German were published using the online tool Google Forms and were completed by 7915 individuals, whereas the final analysis was conducted on 5893 subjects (Figure 1). Participants were excluded due to living abroad, not meeting inclusion criteria about children's age, doubled responses, as well as missing any data. 


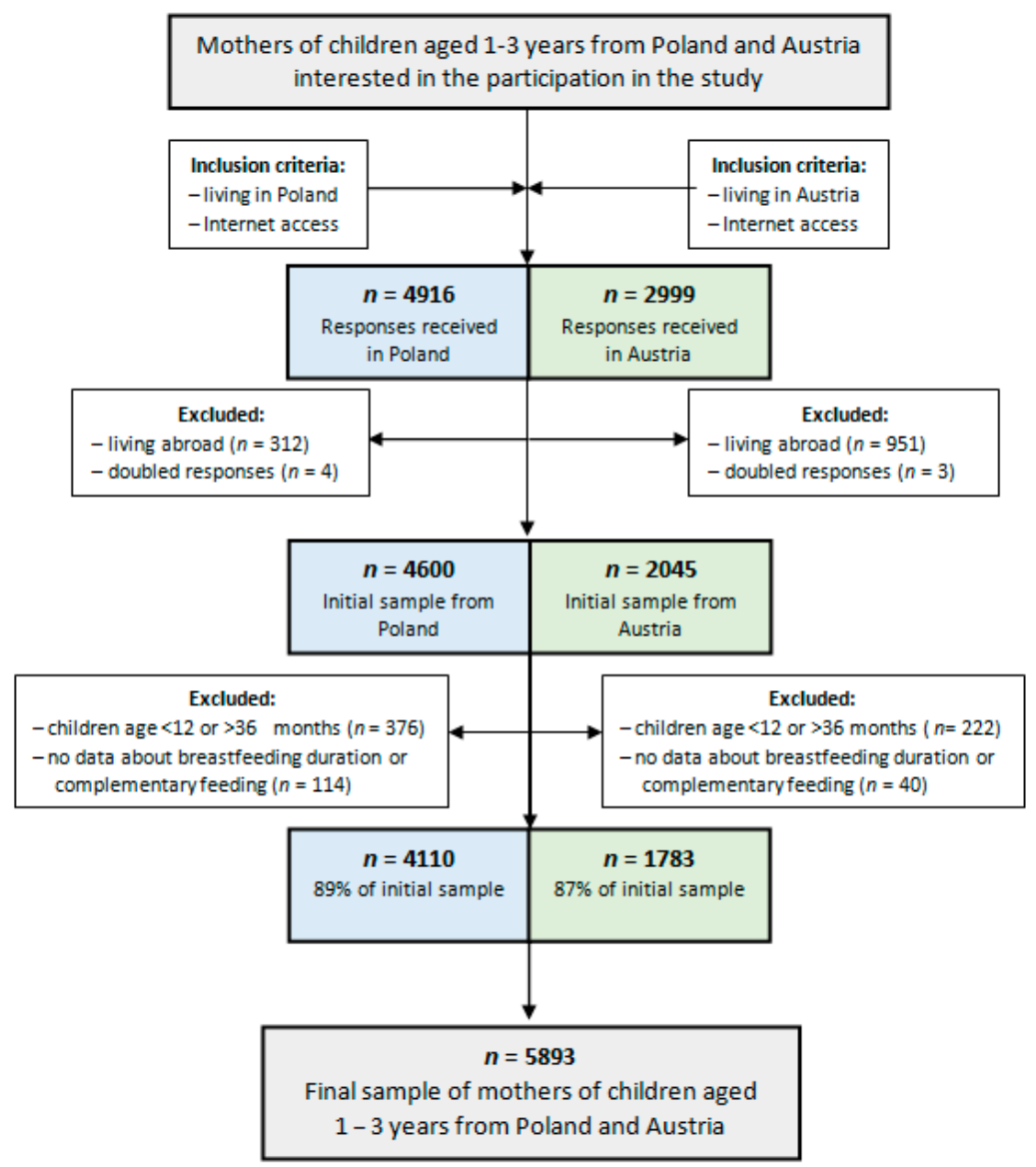

Figure 1. Flowchart of study sample collection.

Detailed study group characteristics from both countries according to age group are presented in Table 1. Most of the mothers from both countries were at least 30 years old, with an average or good economic situation. In most toddlers, solids were introduced between 4 and 6 months of age, using mixed-method and homemade complementary foods. In both countries, mothers of younger toddlers were more often 25-29 years old and primipara. Moreover, younger toddlers were more often currently being breastfed $(p \leq 0.001)$.

Table 1. Study group characteristics according to sociodemographic factors.

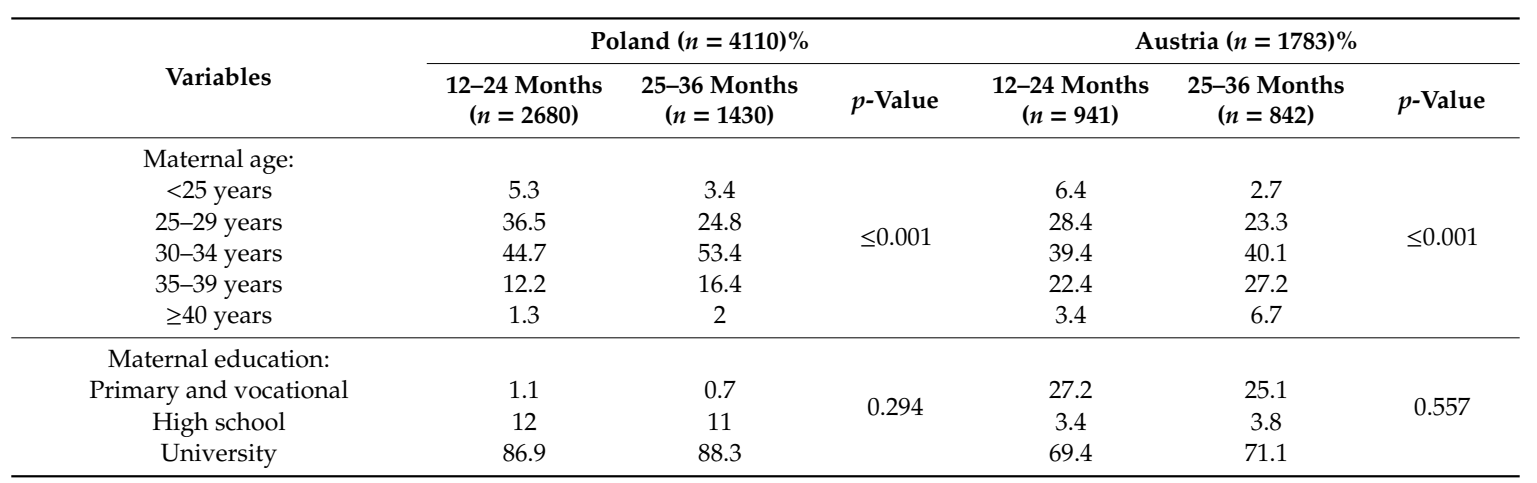


Table 1. Cont.

\begin{tabular}{|c|c|c|c|c|c|c|}
\hline \multirow[b]{2}{*}{ Variables } & \multicolumn{3}{|c|}{ Poland $(n=4110) \%$} & \multicolumn{3}{|c|}{ Austria $(n=1783) \%$} \\
\hline & $\begin{array}{l}\text { 12-24 Months } \\
(n=2680)\end{array}$ & $\begin{array}{l}\text { 25-36 Months } \\
(n=1430)\end{array}$ & $p$-Value & $\begin{array}{l}\text { 12-24 Months } \\
\quad(n=941)\end{array}$ & $\begin{array}{l}\text { 25-36 Months } \\
\quad(n=842)\end{array}$ & $p$-Value \\
\hline 2 persons & 2 & 2 & \multirow{3}{*}{$\leq 0.001$} & 1.5 & 3 & \multirow{3}{*}{$\leq 0.001$} \\
\hline 3 persons & 66.8 & 52.1 & & 55.5 & 36.2 & \\
\hline 4 persons & 23.6 & 38.3 & & 30.9 & 46.3 & \\
\hline \multicolumn{7}{|l|}{ Parity: } \\
\hline Primiparas & 73.3 & 55.8 & \multirow[t]{2}{*}{$\leq 0.001$} & 57.1 & 38.2 & \multirow[t]{2}{*}{$\leq 0.001$} \\
\hline Multiparas & 26.7 & 44.2 & & 42.9 & 61.8 & \\
\hline \multicolumn{7}{|l|}{ Living area: } \\
\hline Rural & 17.5 & 16.9 & 0.601 & 56.5 & 56.1 & 0.839 \\
\hline $51-100 \%$ & 62.3 & 63.2 & \multirow{5}{*}{0.339} & 5.7 & 5.3 & \multirow{5}{*}{0.495} \\
\hline $101-110 \%$ & 23.3 & 24.1 & & 43.6 & 40.3 & \\
\hline $111-130 \%$ & - & - & & 25.8 & 26.7 & \\
\hline $131-150 \%$ & - & - & & 9.3 & 11.3 & \\
\hline$>150 \%$ & - & - & & 15.6 & 16.4 & \\
\hline \multicolumn{7}{|c|}{$\begin{array}{l}\text { Average monthly income per } \\
\text { capita }{ }^{1}:\end{array}$} \\
\hline 1st category & 1.5 & 1.5 & \multirow{4}{*}{0.468} & 8.3 & 8.7 & \multirow{4}{*}{0.008} \\
\hline 2nd category & 15 & 13.5 & & 20.6 & 21 & \\
\hline 3rd category & 32.7 & 32.8 & & 33.5 & 29.3 & \\
\hline 4th category & 17.7 & 16.6 & & 27.5 & 25.1 & \\
\hline \multicolumn{7}{|l|}{ Infant gender: } \\
\hline $4-6$ months & 59.4 & 62.6 & $\leq 0.001$ & 74.9 & 75.5 & 0.878 \\
\hline$\geq 7$ months & 38.6 & 34 & & 20.8 & 20 & \\
\hline Complementary feeding $\mathrm{m}$ & & & & & & \\
\hline TSF & 26.9 & 30.2 & & 38.9 & 33.7 & \\
\hline mixed & 56.6 & 51.1 & 0.004 & 47.7 & 51.4 & 0.076 \\
\hline BLW & 16.5 & 18.7 & & 13.4 & 14.9 & \\
\hline Types of CF: & & & & & & \\
\hline Ready-to-eat foods & 22 & 21.7 & & 22.7 & 24 & \\
\hline Homemade foods & 48.5 & 46.2 & 0.228 & 51.5 & 51.4 & 0.733 \\
\hline Family foods & 29.5 & 32 & & 25.8 & 24.6 & \\
\hline Currently BF: & & & & & & \\
\hline No & 38.8 & 72 & $\leq 0.001$ & 62.4 & 81 & $\leq 0.001$ \\
\hline Yes & 61.2 & 28 & & 37.6 & 19 & \\
\hline EBF duration: & & & & & & \\
\hline Never BF & 5.3 & 5.5 & & 8.1 & 9.5 & \\
\hline Never EBF & 50 & 51.4 & & 32.1 & 28.3 & \\
\hline $1-3$ months & 1.9 & 2.4 & $\leq 0.001$ & 6.3 & 7.5 & 0.506 \\
\hline 4-5 months & 7.8 & 11.3 & & 25.5 & 25.7 & \\
\hline 6-7 months & 34.7 & 28.6 & & 25.5 & 26.7 & \\
\hline$>7$ months & 0.3 & 0.8 & & 2.5 & 2.6 & \\
\hline
\end{tabular}

1. Average monthly income per capita categories depends on country: 1st category $<500$ PLN (Poland)/ $<1000$ EUR (Austria); 2nd category 500-1000 PLN/1000-1500 EUR; 3rd category 1001-2000 PLN/1501-2000 EUR; 4th category 2001-2500 PLN/2001-3000 EUR; 5th category 2501-3000 PLN/3001-5000 EUR; 6th category $\geq 3001$ PLN/ $\geq 5001$ EUR; BF—breastfeeding; BLW—baby-led weaning; CF-complementary foods; CFI—complementary feeding introduction; EBF-exclusive breastfeeding, TSF-traditional spoon-feeding. 


\subsection{Questionnaire}

\subsubsection{Demographic Data}

Data about maternal and paternal age and education level, average monthly income per capita in the household, number of adults and children living in the household, as well as maternal marital status were collected. Moreover, information about the living area, both size of place of residence (recategorized as rural or urban), as well as the region of the country (which was further categorized according to the percentage of EU-28 average gross domestic product (GDP) per capita [32]) was gathered. Additionally, data about children's gender, current age and data about birth outcomes were obtained. Detailed information about collected data was reported previously [31].

\subsubsection{Early Feeding Practices}

Data about the duration of any breastfeeding $(\mathrm{BF})$ and exclusive breastfeeding $(\mathrm{EBF})$, as well as current breastfeeding were collected. Mothers were also asked about formula feeding, including information about giving formula to infants at the maternity ward. According to the World Health Organization (WHO), exclusive breastfeeding was defined as infant feeding with only breastmilk without introducing other foods, drinks or water (except medicines, vitamins or minerals) into the infant diet [33]; therefore, newborns who received formula at the maternity ward were categorized as non-exclusively breastfed. Data about complementary feeding, i.e., timing of introduction (CFI) and method (traditional spoon-feeding (TSF), baby-led weaning (BLW), and partially BLW) of introducing complementary foods were also collected. TSF was defined as solely or mostly spoon-fed by an adult, BLW was defined as baby feeding themselves, and mixed-method was defined as about half spoon-feeding by an adult and half baby feeding themselves. Mothers were also asked about types of complementary foods (CF) that were used during complementary feeding (commercial CF, homemade especially for infant or family foods).

\subsubsection{Toddlers' Dietary Habits}

Mothers were asked about toddlers' feeding behavior during the last 3 months, including the use of AS and ASu. Throughout this paper, phrases "added salt" (AS) and "added sugar" (ASu) refer to the salt and sugar that the parents added during cooking or serving foods for their children. It is not tantamount to the term "added sugars", which is defined by the European Food Safety Authority (EFSA) as "sucrose, fructose, glucose, starch hydrolysates (glucose syrup, high-fructose syrup) and other isolated sugar preparations used as such or added during food preparation and manufacturing". Based on those questions, simultaneous use of AS and ASu was calculated (AS\&Su). Information about frequency of consumption of 17 food items (raw vegetables; cooked vegetables; fruits; natural grain products; sweetened grain products; natural milk products; sweetened milk products; homemade meat dishes and products; processed meat; fish; eggs and egg dishes; legumes; commercial sweets, chocolates and cakes; homemade sweets, chocolates and cakes; fruit or fruit-vegetable juices; vegetable juices; water) was collected according to the following categories (1) never or almost never; (2) less than 1 time per week; (3) 1 time per week; (4) at least 2-4 times per week; (5) 1 time per day; (6) several times per day. Additionally, mothers were asked about methods of consuming meals by their children, their concerns about their toddlers' eating behaviors and sources of knowledge about children's feeding.

\subsection{Statistical Analysis}

All statistical analyses were conducted separately according to both (1) country and (2) age group: 12-24 months or 25-36 months. For nominal variables, results were presented as a percentage, and a chi-square test was performed. Factors associated with the use of AS, ASu and AS\&Su were investigated using univariate and multivariate logistic regression analysis. The models included sociodemographic (maternal age and education, parity, living area, macroeconomic region and average 
monthly income per capita), and early feeding-related variables (CFI, complementary feeding method, types of $\mathrm{CF}$, currently $\mathrm{BF}$ and duration of $\mathrm{EBF}$ ).

Dietary patterns were determined using the $k$-means algorithm separately for both countries but without distinction of age group. Three clusters were selected because they were best interpretable: (1) pro-health, characterized by a high intake of vegetables, fruits, natural milk and grain products, fish, eggs and egg dishes, legumes, water, while low intake of sweetened milk and grain products, homemade and processed meats; (2) Western-like, characterized by a high intake of sweetened milk and grain products, homemade and processed meat, sweets, chocolates and cakes, juices, while low water intake; (3) non-eaters, characterized by lowest or one of the lowest intake of all of the food groups. All analyses were performed using the Statistica 13.3 software (TIBCO Software Inc., Palo Alto, CA, USA). For all analyses, $p \leq 0.05$ was considered significant.

\section{Results}

\subsection{Use of Added Salt, Sugar and Both Salt and Sugar}

In total, AS, ASu and AS\&Su were added into the diet of $58.5 \%, 32.9 \%$, and $26.8 \%$ of toddlers aged 12-24 months (younger group) and 79.2\%, 54.1\%, and 48.5\% aged 25-36 months (older group), respectively (results not presented). Austrian mothers tended to use AS more often than Polish, whereas mothers from Poland were more prone to use ASu and AS\&Su (but only in the older group) more often (Figure 2).
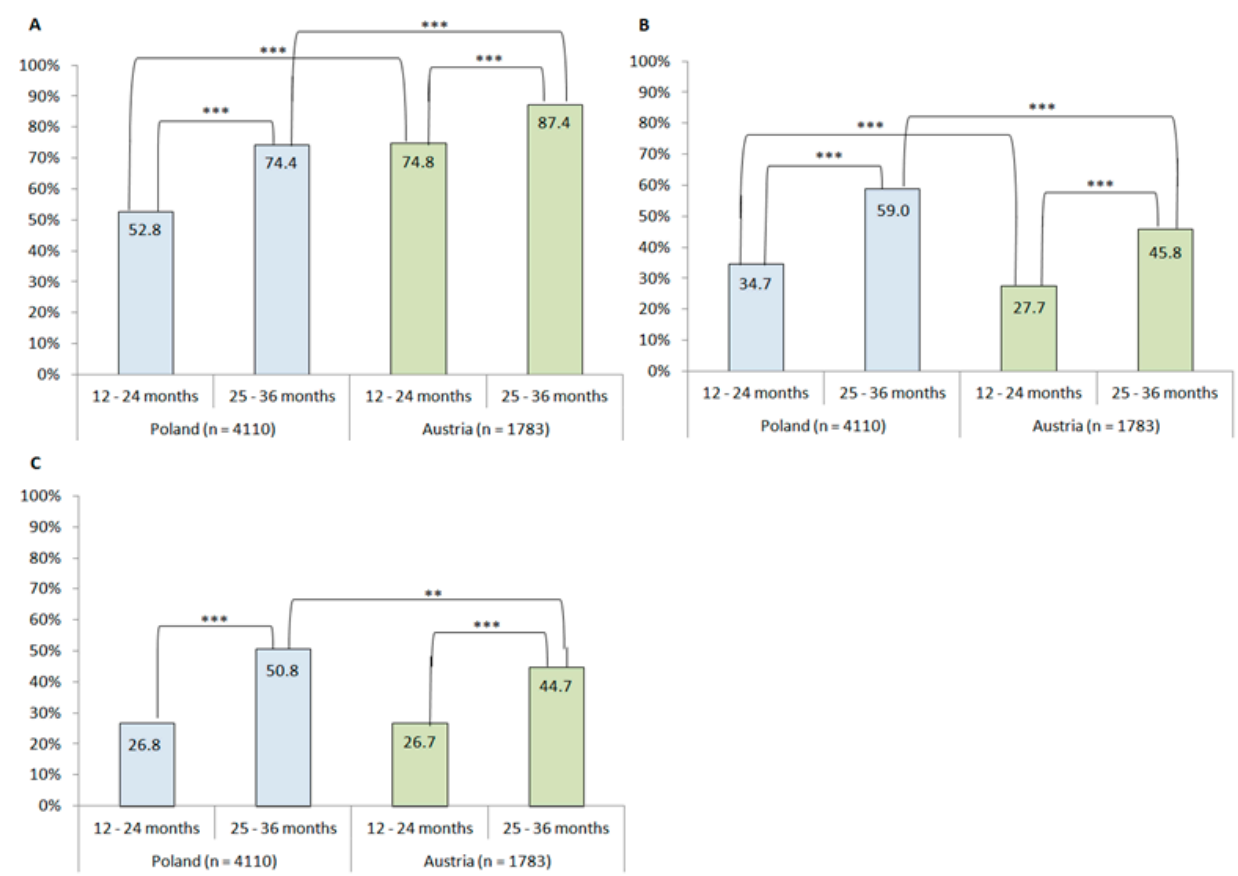

Figure 2. Use of added salt (A), sugar (B), both salt and sugar (C) in toddlers' diets according to country and age group; ${ }^{* *} p \leq 0.01 ;{ }^{* * *} p \leq 0.001$.

\subsection{Factors Associated with the Use of Added Salt, Sugar and Both Salt and Sugar in Toddlers' Diets}

\subsubsection{Sociodemographic Factors}

The results of the multivariate logistic regression analysis conducted in the Polish sample adjusted to sociodemographic and early feeding-related factors are presented in Table 2. The odds of use of AS, ASu, and AS\&Su increased with toddlers' age, but only in the younger group. Factors increasing those odds were multiparty (AS, ASu, and AS\&Su in both age groups), younger maternal age (ASu and AS\&Su in the older group) and moderately high average monthly income (AS and AS\&Su in the 
younger group). Those odds decreased when mothers were older (ASu and AS\&Su in the younger group). In univariate models (Table S1), those odds increased in the group of mothers with lower education (AS and AS\&Su in the younger group), living in a rural area (ASu, AS\&Su in the older group), or mothers with a low monthly income (AS, ASu in the younger group).

Meanwhile, in Austria (Table 3), a multivariate logistic regression model showed that the odds of use of those ingredients increased with toddlers' age (AS, ASu, and AS\&Su in the younger and AS\&Su in the older group). Other factors increasing those odds were maternal multiparity (only in the younger group) and living in a rural area (ASu and AS\&Su in the younger group). Moreover, odds of use of ASu and AS\&Su decreased when mothers were living in the macroeconomic region with the lowest or the highest GDP (older group), but low monthly income increased the odds of use AS (older group). In univariate models (Table S2), those odds increased when the family was living in a rural area (AS in the younger group), or with toddlers' age (ASu in the older group).

\subsubsection{Early Feeding Factors}

A multivariate logistic regression analysis conducted in the Polish sample adjusted to sociodemographic and early feeding-related factors (Table 2) showed that the odds of use of all ingredients were lower when CF were introduced using BLW method (except the use of ASu and AS\&Su in the younger group). Additionally, in the younger group, those odds were higher when introducing CF using the TSF method (AS), when toddlers were never exclusively breastfed (ASu) or were exclusively breastfed for $4-5$ months (AS, ASu, AS\&Su). In the older group, the odds of the use of AS were higher when toddlers were not currently being breastfed and were never breastfed, exclusively breastfed or were exclusively breastfed for $4-5$ months. The univariate models showed that the odds of the use of all ingredients increased in both groups when CFI occurred between 4 and 6 months (except ASu in the younger group). Moreover, in the younger group, those odds increased when mothers used commercial and homemade CF (AS) and toddlers were not currently being breastfed $(\mathrm{AS}, \mathrm{ASu}, \mathrm{AS} \& \mathrm{Su}$ ), were never exclusively breastfed (AS\&Su) or were exclusively breastfed for only 1-3 months (AS).

On the other hand, in Austria, the odds of use of AS, ASu and AS\&Su in the younger group were higher when toddlers were not currently being breastfed (Table 3). In the younger group, EBF for 1-3 months decreased the odds of the use of AS. In the older group, the odds of the use of ASu and AS\&Su were higher when toddlers were never breastfed. Additionally, lack of EBF and EBF for 4-5 months increased the odds of the use of ASu. Univariate models (Table S2) revealed that introducing CF by BLW method decreased the odds (AS in the younger group) and using commercial CF or CFI between 4 and 6 months increased those odds (AS in the older group).

3.3. Current Toddlers' Dietary Habits, Maternal Concerns and Feeding Practices According to the Use of Added Salt, Sugar and Both Salt and Sugar

Polish toddlers in both age groups who had received meals with AS, ASu, and AS\&Su more often followed a Western-like and less pro-health dietary pattern. Moreover, in those groups, more mothers reported concerns about toddlers' eating and poorer feeding practices were observed (Table 4). Meanwhile, in the Austrian sample, similar differences in dietary habits were observed only with the use of ASu and AS\&Su in both age groups (Table 5). 
Table 2. Multivariate regression analysis models of factors influencing the use of added salt, sugar, and salt and sugar in the Polish toddlers $(n=4110)$.

\begin{tabular}{|c|c|c|c|c|c|c|c|}
\hline \multirow{3}{*}{ Factors } & \multirow{3}{*}{ Variable } & \multicolumn{3}{|c|}{ 12-24 Months } & \multicolumn{3}{|c|}{ 25-36 Months } \\
\hline & & \multicolumn{3}{|c|}{$(n=2680)$} & \multicolumn{3}{|c|}{$(n=1430)$} \\
\hline & & AS aOR $(95 \% \mathrm{CI})$ & ASu aOR $(95 \% \mathrm{CI})$ & AS\&Su aOR $(95 \% \mathrm{CI})$ & AS aOR $(95 \% \mathrm{CI})$ & ASu aOR $(95 \% \mathrm{CI})$ & AS\&Su aOR $(95 \% \mathrm{CI})$ \\
\hline \multirow{7}{*}{$\begin{array}{l}\text { Sociodemographic } \\
\text { Factors }\end{array}$} & Toddler age: & $1.18(1.15-1.21)^{* * *}$ & $1.14(1.11-1.17)^{* * *}$ & $1.16(1.13-1.19)^{* * *}$ & $1.01(0.98-1.05)$ & $1.02(0.99-1.05)$ & $1.01(0.98-1.04)$ \\
\hline & $\begin{array}{l}\text { Maternal age: } \\
<25 \text { years } \\
25-29 \text { years } \\
30-34 \text { years } \\
35-39 \text { years } \\
\geq 40 \text { years }\end{array}$ & $\begin{array}{c}0.91(0.61-1.38) \\
1.01(0.84-1.22) \\
1 \\
0.98(0.75-1.28) \\
0.59(0.28-1.23)\end{array}$ & $\begin{array}{c}1.34(0.89-2.02) \\
1.11(0.91-1.34) \\
1 \\
0.79(0.60-1.05) \\
0.20(0.07-0.59)^{* *}\end{array}$ & $\begin{array}{c}1.09(0.70-1.70) \\
1.11(0.91-1.37) \\
1 \\
0.80(0.59-1.08) \\
0.27(0.09-0.83)^{*}\end{array}$ & $\begin{array}{c}1.07(0.48-2.38) \\
1.23(0.90-1.69) \\
1 \\
1.36(0.95-1.94) \\
1.54(0.60-3.94)\end{array}$ & $\begin{array}{c}2.72(1.31-5.65)^{* *} \\
1.49(1.13-1.96)^{* *} \\
1 \\
0.92(0.68-1.25) \\
0.71(0.33-1.52)\end{array}$ & $\begin{array}{c}2.22(1.12-4.37)^{*} \\
1.43(1.09-1.87)^{* *} \\
1 \\
1.11(0.82-1.50) \\
0.88(0.41-1.92)\end{array}$ \\
\hline & $\begin{array}{l}\text { Maternal education: } \\
\text { Primary and vocational } \\
\text { High school } \\
\text { University }\end{array}$ & $\begin{array}{c}2.03(0.83-4.96) \\
1.08(0.83-1.42) \\
1\end{array}$ & $\begin{array}{c}1.39(0.63-3.05) \\
1.04(0.79-1.36) \\
1\end{array}$ & $\begin{array}{c}2.11(0.94-4.72) \\
1.18(0.88-1.57) \\
1\end{array}$ & $\begin{array}{c}2.29(0.28-19.06) \\
1.65(1.03-2.64)^{*} \\
1\end{array}$ & $\begin{array}{c}1.15(0.27-4.85) \\
0.86(0.59-1.26) \\
1\end{array}$ & $\begin{array}{c}1.65(0.40-6.84) \\
1.00(0.69-1.45) \\
1\end{array}$ \\
\hline & $\begin{array}{l}\text { Parity: } \\
\text { Primiparas } \\
\text { Multiparas }\end{array}$ & $\begin{array}{c}1 \\
1.80(1.47-2.20)^{* * * *}\end{array}$ & $\begin{array}{c}1 \\
1.37(1.12-1.67)^{* *}\end{array}$ & $\begin{array}{c}1 \\
1.55(1.26-1.92)^{* * *}\end{array}$ & $\begin{array}{c}1 \\
1.32(1.01-1.71) \text { * }\end{array}$ & $\begin{array}{c}1 \\
1.37(1.09-1.72)^{* *}\end{array}$ & $\begin{array}{c}1 \\
1.38(1.10-1.73)^{* *}\end{array}$ \\
\hline & $\begin{array}{l}\text { Living area: } \\
\text { Rural } \\
\text { Urban }\end{array}$ & $\begin{array}{c}1.02(0.82-1.27) \\
1\end{array}$ & $\begin{array}{c}1.12(0.90-1.40) \\
1\end{array}$ & $\begin{array}{c}1.10(0.87-1.39) \\
1\end{array}$ & $\begin{array}{c}1.08(0.76-1.52) \\
1\end{array}$ & $\begin{array}{c}1.17(0.87-1.58) \\
1\end{array}$ & $\begin{array}{c}1.15(0.86-1.54) \\
1\end{array}$ \\
\hline & $\begin{array}{l}\text { Macroeconomic region }(\mathrm{G} \\
47-50 \% \\
51-100 \% \\
101-110 \% \\
\end{array}$ & $\begin{array}{c}\text { P EU-28 average): } \\
1.07(0.81-1.42) \\
0.99(0.80-1.21) \\
1\end{array}$ & $\begin{array}{c}0.83(0.62-1.11) \\
0.93(0.76-1.15) \\
1\end{array}$ & $\begin{array}{c}0.79(0.57-1.07) \\
0.86(0.69-1.07) \\
1\end{array}$ & $\begin{array}{c}0.86(0.55-1.34) \\
0.98(0.73-1.33) \\
1\end{array}$ & $\begin{array}{c}1.05(0.71-1.55) \\
1.13(0.87-1.47) \\
1\end{array}$ & $\begin{array}{c}0.95(0.65-1.39) \\
1.11(0.86-1.44) \\
1\end{array}$ \\
\hline & $\begin{array}{l}\text { Average monthly income } \\
<500 \\
500-1000 \\
1001-2000 \\
2001-2500 \\
2501-3000 \\
\geq 3001\end{array}$ & $\begin{array}{c}\text { Cr capita (PLN): } \\
1.35(0.64-2.84) \\
1.26(0.90-1.75) \\
1.13(0.85-1.49) \\
1.40(1.03-1.90)^{*} \\
1 \\
0.93(0.69-1.25)\end{array}$ & $\begin{array}{c}0.94(0.45-2.00) \\
1.30(0.92-1.82) \\
1.15(0.86-1.53) \\
1.36(0.99-1.86) \\
1 \\
0.94(0.69-1.28)\end{array}$ & $\begin{array}{c}1.55(0.71-3.37) \\
1.62(1.12-2.36)^{*} \\
1.30(0.94-1.80) \\
1.71(1.21-2.42)^{* *} \\
1 \\
1.09(0.77-1.55)\end{array}$ & $\begin{array}{c}2.23(0.61-8.08) \\
1.16(0.71-1.89) \\
1.17(0.79-1.73) \\
1.32(0.84-2.06) \\
1 \\
1.20(0.79-1.81)\end{array}$ & $\begin{array}{c}1.67(0.57-4.86) \\
0.85(0.55-1.32) \\
0.83(0.58-1.19) \\
1.01(0.67-1.51) \\
1 \\
0.88(0.61-1.28)\end{array}$ & $\begin{array}{c}2.02(0.74-5.54) \\
0.94(0.61-1.44) \\
0.94(0.66-1.34) \\
1.02(0.69-1.51) \\
1 \\
0.87(0.60-1.26)\end{array}$ \\
\hline
\end{tabular}


Table 2. Cont.

\begin{tabular}{|c|c|c|c|c|c|c|c|}
\hline \multirow{3}{*}{ Factors } & \multirow{3}{*}{ Variable } & \multicolumn{3}{|c|}{ 12-24 Months } & \multicolumn{3}{|c|}{ 25-36 Months } \\
\hline & & \multicolumn{3}{|c|}{$(n=2680)$} & \multicolumn{3}{|c|}{$(n=1430)$} \\
\hline & & AS aOR $(95 \% \mathrm{CI})$ & ASu aOR $(95 \% \mathrm{CI})$ & AS\&Su aOR $(95 \%$ CI $)$ & AS aOR $(95 \% \mathrm{CI})$ & ASu aOR $(95 \% \mathrm{CI})$ & AS\&Su aOR $(95 \%$ CI $)$ \\
\hline \multirow{5}{*}{$\begin{array}{l}\text { Feeding-Related } \\
\text { Factors }\end{array}$} & $\begin{array}{l}\text { Timing of CFI: } \\
<4 \text { months } \\
4-6 \text { months } \\
\geq 7 \text { months }\end{array}$ & $\begin{array}{c}1.24(0.51-1.74) \\
1.09(0.91-1.32) \\
1\end{array}$ & $\begin{array}{c}1.01(0.55-1.85) \\
0.94(0.78-1.14) \\
1\end{array}$ & $\begin{array}{c}0.82(0.42-1.59) \\
1.04(0.84-1.28) \\
1\end{array}$ & $\begin{array}{c}1.31(0.57-2.97) \\
1.14(0.86-1.52) \\
1\end{array}$ & $\begin{array}{c}0.69(0.36-1.33) \\
1.10(0.85-1.43) \\
1\end{array}$ & $\begin{array}{c}0.80(0.41-1.54) \\
1.20(0.93-1.54) \\
1\end{array}$ \\
\hline & $\begin{array}{l}\text { Complementary } \mathrm{f} \\
\text { TSF } \\
\text { Mixed } \\
\text { BLW }\end{array}$ & $\begin{array}{l}\text { thod: } \\
1.28(1.04-1.58)^{*} \\
1 \\
0.70(0.55-0.90)^{* *}\end{array}$ & $\begin{array}{c}0.95(0.77-1.17) \\
1 \\
0.95(0.74-1.22)\end{array}$ & $\begin{array}{c}1.03(0.82-1.30) \\
1 \\
0.81(0.61-1.08)\end{array}$ & $\begin{array}{c}1.06(0.77-1.45) \\
1 \\
0.69(0.49-0.98) *\end{array}$ & $\begin{array}{c}1.09(0.83-1.44) \\
1 \\
0.61(0.44-0.83) * *\end{array}$ & $\begin{array}{c}1.06(0.81-1.39) \\
1 \\
0.63(0.46-0.87) * *\end{array}$ \\
\hline & $\begin{array}{l}\text { Types of CF: } \\
\text { Commercial } \\
\text { Homemade } \\
\text { Family }\end{array}$ & $\begin{array}{c}1.18(0.90-1.54) \\
1.01(0.82-1.25) \\
1\end{array}$ & $\begin{array}{c}0.79(0.60-1.03) \\
0.93(0.75-1.16) \\
1\end{array}$ & $\begin{array}{c}0.88(0.66-1.19) \\
1.04(0.82-1.32) \\
1\end{array}$ & $\begin{array}{c}0.60(0.40-1.34) \\
0.73(0.53-1.01) \\
1\end{array}$ & $\begin{array}{c}0.74(0.52-1.06) \\
0.80(0.60-1.06) \\
1\end{array}$ & $\begin{array}{c}0.76(0.53-1.08) \\
0.78(0.59-1.03) \\
1\end{array}$ \\
\hline & $\begin{array}{l}\text { Currently BF: } \\
\text { No } \\
\text { Yes }\end{array}$ & $\begin{array}{c}1.08(0.89-1.30) \\
1\end{array}$ & $\begin{array}{c}1.02(0.84-1.24) \\
1\end{array}$ & $\begin{array}{c}1.02(0.83-1.26) \\
1\end{array}$ & $\begin{array}{c}1.71(1.27-2.29)^{* * *} \\
1\end{array}$ & $\begin{array}{c}0.96(0.73-1.27) \\
1\end{array}$ & $\begin{array}{c}1.15(0.88-1.51) \\
1\end{array}$ \\
\hline & $\begin{array}{l}\text { EBF duration: } \\
\text { Never BF } \\
\text { Never EBF } \\
1-3 \text { months } \\
4-5 \text { months } \\
6-7 \text { months } \\
>7 \text { months }\end{array}$ & $\begin{array}{c}1.06(0.70-1.60) \\
1.04(0.87-1.26) \\
1.77(0.93-3.36) \\
1.63(1.15-2.32)^{* *} \\
1 \\
0.61(0.14-2.69)\end{array}$ & $\begin{array}{c}1.65(1.10-2.48)^{*} \\
1.11(0.91-1.34) \\
0.92(0.48-1.76) \\
1.86(1.33-2.60)^{* * *} \\
1 \\
0.17(0.02-1.46)\end{array}$ & $\begin{array}{c}1.46(0.94-2.27) \\
1.12(0.91-1.38) \\
1.28(0.67-2.48) \\
1.82(1.28-2.59)^{* * *} \\
1 \\
0.26(0.03-2.28)\end{array}$ & $\begin{array}{c}3.50(1.69-7.27)^{* * *} \\
1.34(1.01-1.79)^{*} \\
1.35(0.56-3.26) \\
1.65(1.01-2.69)^{*} \\
1 \\
0.77(0.22-2.76)\end{array}$ & $\begin{array}{c}1.16(0.65-2.09) \\
1.16(0.90-1.51) \\
1.05(0.49-2.23) \\
1.01(0.67-1.52) \\
1 \\
0.98(0.29-3.34)\end{array}$ & $\begin{array}{c}1.47(0.83-2.60) \\
1.20(0.92-1.55) \\
1.33(0.63-2.81) \\
1.10(0.74-1.65) \\
1 \\
1.02(0.30-3.46)\end{array}$ \\
\hline
\end{tabular}

aOR—adjusted odds ratio; AS—added salt; ASu—added sugar; AS\&Su—added salt and sugar; BF—breastfeeding; BLW—baby-led weaning; CF-complementary foods; CFI—complementary feeding introduction; CI—confidence interval; CF-complementary foods; EBF-exclusive breastfeeding; GDP-Gross Domestic Product; TSF-traditional spoon-feeding; ${ }^{*} p \leq 0.05 ;{ }^{* *} p \leq 0.01 ;{ }^{* *} p \leq 0.001$. 
Table 3. Multivariate regression analysis models of factors influencing the use of added salt, sugar, and salt and sugar in the Austrian toddlers $(n=1783)$.

\begin{tabular}{|c|c|c|c|c|c|c|c|}
\hline \multirow{2}{*}{ Factors } & \multirow{2}{*}{ Variable } & \multicolumn{3}{|c|}{ 12-24 Months $(n=941)$} & \multicolumn{3}{|c|}{ 25-36 Months $(n=842)$} \\
\hline & & $\mathrm{AS}$ aOR $(95 \% \mathrm{CI})$ & ASu aOR $(95 \% \mathrm{CI})$ & AS\&Su aOR $(95 \% \mathrm{CI})$ & AS aOR $(95 \% \mathrm{CI})$ & ASu aOR $(95 \% \mathrm{CI})$ & AS\&Su aOR $(95 \% \mathrm{CI})$ \\
\hline \multirow{7}{*}{$\begin{array}{l}\text { Sociodemographic } \\
\text { Factors }\end{array}$} & Toddler age: & $1.16(1.11-1.21)^{* * *}$ & $1.13(1.08-1.18)^{* * *}$ & $1.13(1.08-1.18)^{* * *}$ & $1.03(0.97-1.09)$ & $1.04(1.00-1.08)$ & $1.04(1.00-1.08)^{*}$ \\
\hline & $\begin{array}{l}\text { Maternal age: } \\
<25 \text { years } \\
25-29 \text { years } \\
30-34 \text { years } \\
35-39 \text { years } \\
\geq 40 \text { years }\end{array}$ & $\begin{array}{c}1.67(0.78-3.57) \\
1.15(0.78-1.71) \\
1 \\
0.96(0.63-1.46) \\
0.77(0.32-1.85) \\
\end{array}$ & $\begin{array}{c}1.23(0.64-2.37) \\
1.11(0.76-1.62) \\
1 \\
0.88(0.58-1.33) \\
0.85(0.36-2.01) \\
\end{array}$ & $\begin{array}{c}1.27(0.66-2.46) \\
1.08(0.74-1.58) \\
1 \\
0.89(0.58-1.36) \\
0.92(0.39-2.19)\end{array}$ & $\begin{array}{c}0.55(0.16-1.82) \\
0.73(0.42-1.28) \\
1 \\
0.89(0.52-1.54) \\
0.64(0.28-1.50) \\
\end{array}$ & $\begin{array}{c}0.68(0.28-1.69) \\
0.80(0.55-1.15) \\
1 \\
0.97(0.68-1.38) \\
1.20(0.66-2.16)\end{array}$ & $\begin{array}{c}0.62(0.24-1.55) \\
0.82(0.57-1.19) \\
1 \\
0.94(0.66-1.34) \\
1.15(0.64-2.08) \\
\end{array}$ \\
\hline & $\begin{array}{l}\text { Maternal education: } \\
\text { Primary and vocational } \\
\text { High school } \\
\text { University }\end{array}$ & $\begin{array}{c}1.07(0.73-1.59) \\
0.43(0.19-1.05) \\
1\end{array}$ & $\begin{array}{c}1.08(0.76-1.54) \\
0.65(0.26-1.61) \\
1\end{array}$ & $\begin{array}{c}1.11(0.77-1.59) \\
0.56(0.22-1.46) \\
1\end{array}$ & $\begin{array}{c}0.85(0.50-1.43) \\
1.58(0.34-7.28) \\
1\end{array}$ & $\begin{array}{c}1.08(0.76-1.53) \\
1.56(0.73-3.35) \\
1\end{array}$ & $\begin{array}{c}1.03(0.73-1.47) \\
1.44(0.67-3.08) \\
1\end{array}$ \\
\hline & $\begin{array}{l}\text { Parity: } \\
\text { Primiparias } \\
\text { Multiparias }\end{array}$ & $\begin{array}{c}1 \\
1.61(1.14-2.28)^{* *}\end{array}$ & $\begin{array}{c}1 \\
1.84(1.34-2.54)^{* * *}\end{array}$ & $\begin{array}{c}1 \\
1.85(1.34-2.55)^{* * *}\end{array}$ & $\begin{array}{c}1 \\
0.82(0.52-1.30)\end{array}$ & $\begin{array}{c}1 \\
1.09(0.80-1.47)\end{array}$ & $\begin{array}{c}1 \\
1.11(0.82-1.50)\end{array}$ \\
\hline & $\begin{array}{l}\text { Living area: } \\
\text { Rural } \\
\text { Urban }\end{array}$ & $\begin{array}{c}1.23(0.85-1.78) \\
1\end{array}$ & $\begin{array}{c}1.57(1.09-2.27)^{*} \\
1\end{array}$ & $\begin{array}{c}1.54(1.06-2.24)^{*} \\
1\end{array}$ & $\begin{array}{c}0.96(0.58-1.59) \\
1\end{array}$ & $\begin{array}{c}1.13(0.81-1.58) \\
1\end{array}$ & $\begin{array}{c}1.08(0.77-1.51) \\
1\end{array}$ \\
\hline & $\begin{array}{l}\text { Macroeconomic region }( \\
47-50 \% \\
51-100 \% \\
101-110 \% \\
111-130 \% \\
131-150 \% \\
>150 \%\end{array}$ & $\begin{array}{c}\text { P EU-28 average): } \\
- \\
1.17(0.55-2.48) \\
1 \\
0.85(0.58-1.26) \\
1.16(0.64-2.12) \\
0.82(0.50-1.34)\end{array}$ & $\begin{array}{c}- \\
1.43(0.75-2.73) \\
1 \\
1.35(0.93-1.96) \\
0.69(0.37-1.27) \\
1.31(0.78-2.21)\end{array}$ & $\begin{array}{c}- \\
1.52(0.79-2.91) \\
1 \\
1.31(0.89-1.91) \\
0.67(0.36-1.25) \\
1.25(0.74-2.11)\end{array}$ & $\begin{array}{c}- \\
3.97(0.89-17.66) \\
1 \\
1.16(0.69-1.94) \\
2.06(0.86-4.94) \\
0.95(0.49-1.85)\end{array}$ & $\begin{array}{c}- \\
0.36(0.18-0.72)^{* *} \\
1 \\
0.81(0.57-1.15) \\
0.63(0.38-1.02) \\
0.61(0.38-0.98)^{*}\end{array}$ & $\begin{array}{c}- \\
0.38(0.19-0.76)^{* *} \\
1 \\
0.78(0.55-1.10) \\
0.62(0.38-1.01) \\
0.58(0.36-0.93)^{*}\end{array}$ \\
\hline & $\begin{array}{l}\text { Average monthly income } \\
<1000 \\
1000-1500 \\
1501-2000 \\
2001-3000 \\
3001-5000 \\
\geq 5001\end{array}$ & $\begin{array}{c}\text { cr capita (EUR): } \\
0.80(0.37-1.70) \\
1.05(0.55-2.02) \\
1.15(0.63-2.10) \\
1.04(0.56-1.92) \\
1 \\
0.84(0.22-3.17)\end{array}$ & $\begin{array}{c}1.00(0.46-2.17) \\
1.56(0.82-2.97) \\
0.99(0.54-1.82) \\
1.21(0.65-2.24) \\
1 \\
1.59(0.44-5.75)\end{array}$ & $\begin{array}{c}1.27(0.57-2.82) \\
1.78(0.91-3.48) \\
1.26(0.66-2.38) \\
1.46(0.76-2.78) \\
1 \\
1.95(0.53-7.10)\end{array}$ & $\begin{array}{c}1.53(0.65-3.62) \\
2.45(1.16-5.18)^{*} \\
1.55(0.81-2.97) \\
1.60(0.82-3.15) \\
1 \\
1.58(0.41-6.06)\end{array}$ & $\begin{array}{c}0.95(0.51-1.78) \\
1.22(0.74-2.02) \\
1.14(0.71-1.83) \\
1.10(0.68-1.80) \\
1 \\
1.41(0.57-3.47)\end{array}$ & $\begin{array}{c}0.89(0.47-1.66) \\
1.21(0.73-2.01) \\
1.15(0.71-1.85) \\
1.10(0.68-1.79) \\
1 \\
1.22(0.50-3.01)\end{array}$ \\
\hline
\end{tabular}


Table 3. Cont

\begin{tabular}{|c|c|c|c|c|c|c|c|}
\hline \multirow{2}{*}{ Factors } & \multirow{2}{*}{ Variable } & \multicolumn{3}{|c|}{ 12-24 Months $(n=941)$} & \multicolumn{3}{|c|}{ 25-36 Months $(n=842)$} \\
\hline & & AS aOR $(95 \% \mathrm{CI})$ & ASu aOR $(95 \% \mathrm{CI})$ & AS\&Su aOR $(95 \% \mathrm{CI})$ & AS aOR $(95 \% \mathrm{CI})$ & ASu aOR $(95 \% \mathrm{CI})$ & AS\&Su aOR $(95 \% \mathrm{CI})$ \\
\hline \multirow{21}{*}{$\begin{array}{l}\text { Feeding-Related } \\
\text { Factors }\end{array}$} & Timing of CFI: & & & & & & \\
\hline & $<4$ months & $1.04(0.42-2.55)$ & $0.57(0.24-1.36)$ & $0.55(0.22-1.34)$ & $1.75(0.51-5.97)$ & $0.93(0.43-2.02)$ & $0.80(0.37-1.75)$ \\
\hline & $4-6$ months & $1.14(0.74-1.76)$ & $0.91(0.59-1.40)$ & $0.91(0.58-1.40)$ & $1.66(0.93-2.96)$ & $0.90(0.60-1.35)$ & $0.89(0.59-1.34)$ \\
\hline & $\geq 7$ months & 1 & 1 & 1 & 1 & 1 & 1 \\
\hline & Complementary $\mathrm{f}$ & thod: & & & & & \\
\hline & TSF & $0.87(0.60-1.26)$ & $1.19(0.83-1.70)$ & $1.13(0.79-1.62)$ & $1.26(0.75-2.10)$ & $0.97(0.70-1.34)$ & $0.92(0.66-1.27)$ \\
\hline & Mixed & 1 & 1 & 1 & 1 & 1 & 1 \\
\hline & BLW & $0.61(0.35-1.05)$ & $0.70(0.39-1.25)$ & $0.69(0.38-1.24)$ & $0.74(0.39-1.40)$ & $0.89(0.56-1.43)$ & $0.81(0.51-1.31)$ \\
\hline & Types of CF: & & & & & & \\
\hline & Commercial & $1.15(0.65-2.04)$ & $0.82(0.48-1.38)$ & $0.94(0.55-1.59)$ & $1.39(0.66-2.95)$ & $0.78(0.48-1.26)$ & $0.84(0.52-1.35)$ \\
\hline & Homemade & $0.82(0.51-1.33)$ & $0.73(0.46-1.14)$ & $0.81(0.51-1.27)$ & $0.79(0.44-1.40)$ & $0.81(0.54-1.21)$ & $0.81(0.54-1.21)$ \\
\hline & Family & 1 & 1 & 1 & 1 & 1 & 1 \\
\hline & $\begin{array}{l}\text { Currently BF: } \\
\text { No }\end{array}$ & $1.51(1.01-2.25)^{*}$ & $1.56(1.03-2.37)$ * & $1.63(1.07-2.49)$ * & $1.52(0.78-2.94)$ & $1.24(0.74-2.09)$ & $1.36(0.80-2.29)$ \\
\hline & Yes & 1 & 1 & 1 & 1 & 1 & 1 \\
\hline & EBF duration: & & & & & & \\
\hline & Never BF & $0.92(0.46-1.82)$ & $1.52(0.75-3.09)$ & $1.52(0.74-3.09)$ & $1.92(0.62-5.93)$ & $2.39(1.16-4.95)^{*}$ & $2.30(1.11-4.79)$ * \\
\hline & Never EBF & $1.47(0.93-2.34)$ & $1.46(0.94-2.26)$ & $1.31(0.84-2.04)$ & $0.76(0.41-1.39)$ & $1.53(1.01-2.32) *$ & $1.42(0.94-2.16)$ \\
\hline & $1-3$ months & $0.44(0.21-0.88)^{*}$ & $0.87(0.42-1.78)$ & $0.63(0.30-1.34)$ & $0.62(0.25-1.55)$ & $1.66(0.90-3.06)$ & $1.42(0.77-2.63)$ \\
\hline & $4-5$ months & $0.92(0.57-1.47)$ & $1.00(0.62-1.61)$ & $0.93(0.58-1.50)$ & $0.79(0.41-1.50)$ & $1.53(1.00-2.35)^{*}$ & $1.45(0.94-2.22)$ \\
\hline & 6-7 months & 1 & 1 & 1 & 1 & 1 & 1 \\
\hline & $>7$ months & $1.81(0.60-5.47)$ & $1.67(0.62-4.51)$ & $1.78(0.66-4.83)$ & $0.62(0.20-1.86)$ & $1.47(0.57-3.75)$ & $1.51(0.59-3.87)$ \\
\hline
\end{tabular}

CFI-complementary feeding introduction. CI-confidence interval. CF-complementary foods; EBF-exclusive breastfeeding. GDP-Gross Domestic Product; TSF-traditional spoon-feeding; ${ }^{*} \leq \leq 0.05 ; * * \leq 0.01 ; * * * 0.001$. 
Table 4. Current toddlers' dietary habits and maternal concerns about toddlers' feeding according to the age group and use of added salt, sugar, and both salt and sugar in the Polish sample $(n=4110)$.

\begin{tabular}{|c|c|c|c|c|c|c|c|c|c|c|c|c|}
\hline \multirow{3}{*}{ Variables } & \multicolumn{6}{|c|}{ 12-24 Months $(n=2680)$} & \multicolumn{6}{|c|}{ 25-36 Months $(n=1430)$} \\
\hline & \multicolumn{2}{|c|}{ AS } & \multicolumn{2}{|c|}{ ASu } & \multicolumn{2}{|c|}{ AS\&Su } & \multicolumn{2}{|c|}{ AS } & \multicolumn{2}{|c|}{ ASu } & \multicolumn{2}{|c|}{ AS\&Su } \\
\hline & Yes $52.8 \%$ & No $47.2 \%$ & Yes $34.7 \%$ & No $65.3 \%$ & Yes $26.8 \%$ & No $73.2 \%$ & Yes $74.4 \%$ & No $25.6 \%$ & Yes $59.0 \%$ & No $41.0 \%$ & Yes $50.8 \%$ & No $49.2 \%$ \\
\hline \multicolumn{13}{|l|}{ Dietary pattern: } \\
\hline$\rightarrow$ Pro-health & 35.1 & $54.6^{* * *}$ & 37 & $48.1^{* * *}$ & 31.6 & $48.9^{* * *}$ & 28.9 & $44.8^{* * *}$ & 32.6 & $40.9^{* * *}$ & 26.6 & $39.5^{* * *}$ \\
\hline$\rightarrow$ Western-like & 35.3 & 14.2 & 38.6 & 18.3 & 43.3 & 18.8 & 55.4 & 35.5 & 42.5 & 22.4 & 59.8 & 40.5 \\
\hline$\rightarrow$ Non-eaters & 29.6 & 31.1 & 24.3 & 33.5 & 25.1 & 32.3 & 15.8 & 19.7 & 24.9 & 36.8 & 13.6 & 20 \\
\hline Eating at least one meal with the family & 97.5 & 97.1 & 98.4 & $96.7^{* *}$ & 98.2 & 97.0 & 98.3 & 97.3 & 99.2 & $99.3^{* *}$ & 98.6 & 97.4 \\
\hline Eating meals separately & 50.2 & 49.4 & 46.7 & $51.5^{*}$ & 47.2 & 50.8 & 51.6 & $44.8^{*}$ & 35.2 & $41.5^{*}$ & 52.6 & $47.0^{*}$ \\
\hline Eating while watching TV ${ }^{1}$ & 34.1 & $19.8^{* * *}$ & 32.9 & $24.4^{* * *}$ & 37.2 & $23.8^{* * *}$ & 49.3 & $36.3^{* * *}$ & 13.4 & $8.5^{* * *}$ & 51.5 & $40.3^{* * *}$ \\
\hline Eating during playing & 9.9 & 9.1 & 11.7 & $8.3^{* *}$ & 10.9 & 9.0 & 12.3 & 12.6 & 12.7 & 11.9 & 12.3 & 12.5 \\
\hline None concern & 70.8 & $76.8^{* * *}$ & 70.7 & $75.2^{* *}$ & 69.1 & $75.3^{* * *}$ & 68.4 & $76.2^{* *}$ & 67.6 & $74.4^{* *}$ & 65.8 & $75.1^{* * *}$ \\
\hline Toddler eats too little & 17.6 & $13.9^{* *}$ & 17.3 & 15.1 & 18.9 & $14.7^{* *}$ & 14.8 & 11.5 & 14.6 & 12.9 & 15.8 & 11.9 \\
\hline Toddler eats too much & 7.2 & 6.9 & 7.3 & 6.9 & 7.1 & 7.0 & 4.5 & 4.4 & 4.2 & 4.9 & 4.0 & 5.0 \\
\hline Toddler eats same thing for longer period & 20.2 & $15.7^{* *}$ & 20.8 & $16.7^{* *}$ & 22.3 & $16.6^{* * *}$ & 25.8 & 21.9 & 26.1 & 23.0 & 27.4 & $22.2^{*}$ \\
\hline Toddler does not eat vegetables & 19.4 & $11.9^{* * *}$ & 18.6 & $14.4^{* *}$ & 20.6 & $14.1^{* * *}$ & 25.5 & $18.3^{* * *}$ & 26.0 & $20.3^{* *}$ & 27.1 & $20.0^{* *}$ \\
\hline Toddler does not eat novel foods & 16.3 & $11.5^{* * *}$ & 16.0 & $13.0 *$ & 17.4 & $12.8^{* *}$ & 27.6 & $21.6^{*}$ & 28.6 & $22.5^{* *}$ & 29.9 & $22.2^{* * *}$ \\
\hline
\end{tabular}

${ }^{1}$ including smartphones and tablets; AS—added salt; ASu—added sugar; AS\&Su—added salt and sugar; ${ }^{*} p \leq 0.05 ;{ }^{* *} p \leq 0.01 ;{ }^{* * *} p \leq 0.001$. 
Table 5. Current toddlers' dietary habits and maternal concerns about toddlers' feeding according to the age group and use of added salt, sugar, and both salt and sugar in the Austrian sample $(n=1783)$.

\begin{tabular}{|c|c|c|c|c|c|c|c|c|c|c|c|c|}
\hline \multirow{3}{*}{ Variables } & \multicolumn{6}{|c|}{ 12-24 Months $(n=941)$} & \multicolumn{6}{|c|}{ 25-36 Months $(n=842)$} \\
\hline & \multicolumn{2}{|c|}{ AS } & \multicolumn{2}{|c|}{ ASu } & \multicolumn{2}{|c|}{ AS\&Su } & \multicolumn{2}{|c|}{ AS } & \multicolumn{2}{|c|}{ ASu } & \multicolumn{2}{|c|}{ AS\&Su } \\
\hline & Yes $74.8 \%$ & No $25.2 \%$ & Yes $27.7 \%$ & No $72.3 \%$ & Yes $26.8 \%$ & No $73.2 \%$ & Yes $87.4 \%$ & No $12.6 \%$ & Yes $45.8 \%$ & No $54.2 \%$ & Yes $44.7 \%$ & No $55.3 \%$ \\
\hline \multicolumn{13}{|l|}{ Dietary pattern: } \\
\hline$\rightarrow$ Pro-health & 37.8 & $40.9^{* * *}$ & 32.6 & $40.9^{* * *}$ & 33.1 & $40.6^{* * *}$ & 28.8 & 38.7 & 24.6 & $34.6^{* * *}$ & 25 & $34.1^{* * *}$ \\
\hline$\rightarrow$ Western-like & 32.4 & 14.8 & 42.5 & 22.4 & 42.2 & 22.8 & 54.6 & 42.5 & 60.9 & 46.5 & 60.9 & 46.8 \\
\hline$\rightarrow$ Non-eaters & 29.8 & 44.3 & 24.9 & 36.8 & 24.7 & 36.7 & 16.6 & 18.9 & 14.5 & 18.9 & 14,1 & 19.1 \\
\hline Eating at least one meal with the family & 99.4 & 98.7 & 99.2 & 99.3 & 99.2 & 99.3 & 99.5 & 0.0 & 99.7 & 99.3 & 99.7 & 99.4 \\
\hline Eating meals separately & 38.2 & 44.3 & 35.2 & 41.5 & 35.5 & 41.3 & 34.2 & 31.1 & 34.5 & 33.3 & 34.6 & 33.3 \\
\hline Eating while watching TV ${ }^{1}$ & 9.9 & 9.7 & 13.4 & $8.5^{*}$ & 12.7 & 8.8 & 17.8 & 16.0 & 18.9 & 16.4 & 18.6 & 16.7 \\
\hline Eating during playing & 7.5 & 8.4 & 5.7 & 8.5 & 5.6 & 8.6 & 7.1 & 11.3 & 8.0 & 7.2 & 8.0 & 7.3 \\
\hline None concern & 62.8 & 62.9 & 59.4 & 64.1 & 69.6 & 63.6 & 62.9 & 56.6 & 61.7 & 62.5 & 62.2 & 62.0 \\
\hline Toddler eats too little & 17.8 & 16.9 & 20.3 & 16.5 & 19.9 & 16.7 & 19.3 & 17.0 & 20.2 & 18.0 & 18.0 & 20.2 \\
\hline Toddler eats too much & 3.3 & $0.8^{*}$ & 1.9 & 2.9 & 2.0 & 2.9 & 3.3 & 2.8 & 3.9 & 2.6 & 2.6 & 4.0 \\
\hline Toddler eats same thing for longer period & 14.8 & 15.6 & 16.1 & 14.6 & 15.9 & 14.6 & 16.7 & 13.2 & 16.8 & 15.8 & 16.3 & 16.2 \\
\hline Toddler does not eat vegetables & 11.6 & 11.4 & 12.6 & 11.2 & 12.4 & 11.3 & 14.7 & 10.4 & 16.1 & 12.5 & 12.7 & 16.0 \\
\hline Toddler does not eat novel foods & 8.4 & 7.6 & 10.3 & 7.4 & 10.4 & 7.4 & 15.5 & 9.4 & 16.6 & 13.2 & 13.1 & 16.8 \\
\hline
\end{tabular}

${ }^{1}$ including smartphones and tablets; AS—added salt; ASu—added sugar; AS\&Su—added salt and sugar; ${ }^{*} p \leq 0.05 ;{ }^{* *} p \leq 0.01$; *** $p \leq 0.001$. 
3.4. Sources of Knowledge about Children's Feeding According to the Use of Added Salt, Sugar and Both Salt and Sugar

Furthermore, in the present study, associations between sources of knowledge about children's feeding and the use of AS, ASu, and AS\&Su were evaluated (Table S3). Mothers who used those ingredients tended to report more often family and friends (in Austria, only in relation to ASu and AS\&Su in the younger group), but less often nutritionists or dietitians as a source of knowledge acquisition (Poland: AS and AS\&Su in the younger group; Austria: AS, ASu, AS\&Su in the younger and AS in the older group). Moreover, Polish mothers more often indicated magazines (AS in the younger group), TV (AS, ASu, and AS\&Su in the younger group), but less often books, as a source of knowledge acquisition (AS and AS\&Su in the younger group; AS, ASu, and AS\&Su in the older group). Additionally, in the Austrian sample, those mothers who used ASu and AS\&Su more often declared doctors or midwives as their source of knowledge about children's feeding.

\section{Discussion}

The present study demonstrated a high prevalence of the use of added salt, and lower of added sugar and both ingredients in the diets of the Polish and Austrian toddlers. Furthermore, those rates were higher in toddlers aged 25-36 months compared to 12-24 months. It was also observed that the strongest sociodemographic factor associated with the use of added salt, sugar and both salt and sugar was multiparity. Among early feeding factors, current breastfeeding, duration of exclusive breastfeeding and method of introducing complementary foods were the strongest. Moreover, toddlers who consumed meals with added salt and sugar also showed poorer dietary patterns and meal environment. Interestingly, it was also observed that those mothers who used added salt (and sugar to a lesser extent) tended to be more concerned about their children's feeding practices. The obtained results were specific for the country, as well as diminished with toddlers' age.

\subsection{Prevalence of the Use of Added Salt and Sugar in the Toddlers' Diets}

Half of the Polish mothers and three-quarters of the Austrian mothers declared the use of added salt in the diet of their two year old toddlers; for added sugar, these came to one-third for the Polish mothers and one-quarter for the Austrian mothers. Therefore, those rates were higher among older toddlers. Previous studies reported that from 25\% (France [27]) to 50\% (USA [34]) and 60\% (Sri Lanka [35]) of infants received meals with the addition of salt before the end of 12 months of age. A study from Australia showed that around $40 \%$ of toddlers aged 2-3 years consumed meals with added salt, and $11 \%$ of them added salt at the table [24]. Sugar was added into the diet of $25 \%$ of 10 month infants from France [27] and $42 \%$ of infants from Sri Lanka [35]. These rates increased with children's age, which is in accordance with the results of other studies conducted in Europe [27,36,37] and was also confirmed in the present study. The odds of the use of added salt and sugar into toddlers' diets increased by $18 \%$ and $14 \%$ (Poland) and $16 \%$ and $13 \%$ (Austria) per month in the second year of life, respectively. Whereas in the present study, only the use of added salt and sugar as ingredients was assessed, various studies investigated the use of added salt or sugar as well as the intake of salt or sodium and added or free sugars. The use of added salt and sugar intake is inseparably associated with sodium and added/free sugars intake, which also increased with children's age [21-23,28,37-41]. Unfortunately, many infants and toddlers around the world have intakes of sodium $[25,26,38,39,42,43]$ and added/free sugars [22,28,37,39,40,43-45] already close to the upper limit or even above recommendations. Interestingly, many studies conducted on infants and children have shown that boys compared to girls had a higher intake of sodium [21,23,26,46]. Concerning sugar intake, the results are inconclusive. Some studies demonstrated higher intake among girls than boys [40], whereas in the study conducted by Yuan et al. [47], boys were more prone to consume added sugar, however, it was only observed at 8 months of age. Nonetheless, in our study, we did not observe any gender differences in the prevalence of use of added salt, sugar and both salt and sugar (results not presented). 


\subsection{Sociodemographic Factors Associated with the Use of Added Salt and Sugar in the Toddlers' Diets}

Multiparity was associated with the use of added salt and sugar in the toddlers' diets, whereas maternal age, education, and the economic situation showed limited influence. Multiparity was the strongest factor that increased the odds of use of added salt, sugar and both ingredients by $32-85 \%$ in the diets of the Polish toddlers aged 12-36 months and the Austrian toddlers aged 12-24 months. Previously, it was reported that Japanese 3 year old toddlers with older siblings exhibited a higher urinary sodium excretion, as well as daily consumption of snacks compared to firstborn children [48]. Results concerning the use of added sugar and parity are ambiguous, as in the study of Marinho et al. [40], no association between having siblings and use of added sugar was found, but Yuan et al. [47] demonstrated that consumption of added sugar was associated with having a multiparous mother. Our results are consistent with previous results from France [27] and observations from a focus study conducted in five European countries, in which some parents declared following the nutritional guidelines for the firstborn child, but for the second one they "did not follow them as closely" and in consequence, dietary practices were poorer [49,50]. Maternal education remained not significant after adjustment for covariates. Whereas poorer early feeding practices were associated with maternal lower education level [31,51,52], no associations regarding salt use or sodium intake in infants and toddlers [25,26,34] were observed, in contrast to free sugars intake in children [40]. Moreover, a limited inverse association between economic situation and use of added salt and both ingredients in the diet of the Polish toddlers aged 12-24 months, as well as salt in the diet of the Austrian toddlers in this age was noticed. Although a lower economic situation was previously linked to poorer feeding practices during the first years of life [53], it was not observed in relation to sodium [46] or added sugars intake [37], nor sugar and salt use [27], as well as the timing of introducing solids analyzed in our previous study [31].

\subsection{Early Feeding Factors Associated with the Use of Added Salt and Sugar in the Toddlers' Diet}

A lack of current breastfeeding increased the odds of use of added salt, sugar, and both of them in the younger group from Austria, as well as the odds of the use of added salt in the older group from Poland. Additionally, a lack or lower duration of exclusive breastfeeding increased the odds of use of added sugar and both salt and sugar in the younger group, and use of added salt in the older group from Poland, as well as the use of added sugar and both ingredients in the older group from Austria. These results are in agreement with previous studies that linked breastfeeding with better dietary habits during infancy and subsequent life $[23,42,54]$ and lower sodium intake in breastfed children $[25,26,42]$. Nevertheless, in the study of Yuan et al. [47], intake of added sugars was not significantly associated with breastfeeding duration. Breastfeeding is also associated with higher intake of unprocessed foods and lower intake of processed foods, which are main sources of salt/sodium and added sugars in the diet $[23,24,54,55]$. Breastmilk is an important source of chemosensory information and plays a pivotal role in developing children's food preferences [2,8]. Moreover, breastfeeding mothers tend to be older and better educated than those who are not breastfeeding their children $[42,54]$. This fact may lead to better compliance with nutritional guidelines and in consequence, healthier dietary and lifestyle habits in family and children $[2,4,26,51,56,57]$. In contrary to previous results, a study from France found that any breastfeeding duration was positively associated with use of added salt and sugar in infants' diet [27]. The authors hypothesized that it may be caused by a choice of introducing homemade complementary foods rather than commercial ones, which was higher in breastfeeding mothers and was confirmed in other studies [27,54]. Unfortunately, several studies reported that homemade or family foods were often prepared with salt or sugar addition against nutritional recommendations and consequently, contributed into sodium or added sugars intake $[8,10,25,26]$.

In the present study, we did not observe a significant association between the use of added salt or sugar and types of complementary foods. Interestingly, the use of commercial complementary foods tends to be a risk factor of the use of added salt in the younger Polish and older Austrian toddlers. Despite a lack of association in the present study, a previous longitudinal investigation reported that higher consumption of commercial baby foods increased the intake of added sugars 
in later childhood [22]. Analysis of commercial baby foods from four cities in the European region revealed that many of them contained more than the recommended amount of sodium and sugars, possibly due to the addition of salty or sweet ingredients, such as ham, cheese, or concentrated fruit juices [10]. These results are consistent with previous studies from the USA, which showed that $58 \%$ and $45 \%$ [58] or $84 \%$ and $70 \%$ [59] of commercial toddler foods had high sodium or added sugar levels, respectively. The authors emphasized that many commercial infant and toddler foods did not fulfill the criteria of healthy foods in accordance with age, despite parents' perception [49,58].

Timing and method of introducing complementary foods into an infant's diet are other aspects that might determine subsequent dietary habits and food preferences [2,8]. In a few previous studies, it was observed that too early starting of complementary feeding (before 4 months) was related to salt/sodium intake [25-27] and use of added sugar [27]. However, our study did not replicate these findings, as the association between the use of added salt, sugar, and age of introducing complementary foods did not remain significant after adjustment to maternal sociodemographic and early feeding factors. Moreover, early introducing of solids seems to be a plausible risk factor of the use of added salt in toddlers' diet. Parents who introduced solids earlier had more time to introduce salt and sugar [27], and those children were characterized by poorer dietary patterns or feeding difficulties $[56,60]$. This may be partially explained by poorer following of nutritional recommendations [57] and maternal characteristics [31,51,53], similar to breastfeeding.

Finally, the method of introducing complementary foods may be an important factor that contributes to shaping dietary habits and food preferences [61]. We observed that following the BLW method decreased odds of use of added salt in the diet of the Polish toddlers, whereas traditional spoon-feeding increased them in the younger group. Moreover, BLW decreased odds of use of added sugar and both ingredients in the older toddlers. Previous studies have shown equivocal results of sodium intake according to the method of introducing solids. Morison et al. [62] reported no difference in intake of sodium or sugars according to the method of introducing solids, whereas in a study of Erickson et al. [38], infants who were introduced to solids using a modified BLW method (BLISS) had a higher intake of sodium at 7 months, but not at 24 months, with no difference in added sugars intake. Those differences may be caused by higher consumption of family foods than commercial complementary foods during feeding by the BLW method [63]. Our results may be explained in two ways. First, infants who were introduced to solids by the BLW method tend to be breastfed longer, introduced to complementary foods later, and their mothers had better characteristics which lead to healthier feeding practices [31,62-66]. Second, the BLW method is associated with more responsive parental feeding practices [64] and was linked to lower fussiness in later childhood [65].

\subsection{The Use of Added Salt and Sugar and Toddlers' Dietary Habits and Maternal Concerns About Eating}

Interestingly, in the present study, toddlers' dietary patterns varied under consideration of the consumption of meals with or without added salt or sugar. The Polish and Austrian toddlers who consumed added salt and sugar more often followed a Western-like pattern and less often pro-health pattern, characterized by more frequent consumption of low-processed foods, such as vegetables and fruits, grains, or water. Those results are consistent with previous studies that linked salt intake with consumption of sugar-sweetened beverages [67] or consumption of savory snacks with sugar-sweetened beverages and sweets [56]. Toddlers who a followed Western-like dietary pattern may be a risk group of higher sodium/salt $[24,26,39,68,69]$ or sugars $[37,39,40,45]$ intake from processed foods which are characteristic of this pattern. Moreover, higher consumption of salty and sweet foods may blunt the appetite and decrease the intake of nutrient-dense food [35]. Furthermore, early exposure to unhealthy foods is associated with enhanced preference and increased intake of these foods in later childhood $[23,70]$. In the present study, around one-quarter of the younger and half of the older toddlers from both age groups consumed meals with added both salt and sugar. The results are in accordance with previous studies which reported that the introduction of those ingredients into infants' or toddlers' diets is highly associated with each other $[23,27,34,35,71]$. This may be explained in several 
ways. Firstly, salty and sweet preferences in children are correlated with each other and are related to dietary intake of salty, but not sweet foods [72]. Secondly, a lot of commercial complementary foods contain not only too much salt/sodium, but also sugar $[58,59]$. Thirdly, mothers who do not follow strictly nutritional guidelines about salt may be more likely to also add sugar during the complementary feeding period [57]. Furthermore, Polish toddlers, who consumed meals with added salt and sugar, were more often eating meals during watching TV, which may be one of the predictors of poorer dietary habits [56].

Regarding the results considering the mothers' concerns about their toddlers' eating behaviors, it could be noticed that, the Polish mothers who prepared meals without the addition of salt and sugar were less concerned about their toddlers' eating, whereas in other groups, concerns about "eating too little" (only salt and both ingredients), "not eating vegetables" or "not eating novel foods" were reported more often. Therefore, we hypothesized that those mothers may use added salt and sugar as an encouraging strategy to increase the intake of those foods, as mothers who are more concerned about children's eating are prone to have less responsive feeding practices [4]. Furthermore, the addition of salt, but not sugar, has a dose-dependent influence on the toddlers' vegetable intake [73]. However, in a previous study from France, the authors did not observe any association between maternal concerns about the child's health and salt or sugar use during infancy [27].

\subsection{Sources of Knowledge About Children's Feeding}

Presented results showed that mothers of the Polish toddlers who used added salt and sugar in children's meals more often declared family or friends and TV as sources of knowledge about children's feeding, whereas nutritionists or dieticians were reported less often. Similar results were observed previously by Bournez et al. [27] in regard to sugar, but not salt use. However, in both studies, the Internet was the main source of knowledge about nutrition. Previously, relatively high parental consciousness about health risks and the necessity of reduction in salt and sugar in children's feeding were reported [48,71]. Morinaga et al. [48] did not find any associations with salt intake in children, whereas Khokhar et al. [71] reported that those parents more often declared behaviors aimed to reduce salt intake in children. Interestingly, some studies suggest that children of parents who restricted sugar intake have increased sweetness preference [5]. Those results emphasize the necessity of educational interventions to improve parental practices from the beginning of the introduction of solids into the infant's diet.

Most of the results observed in the present study were significant only in the Polish sample, especially the younger group. A potential explanation for this difference between results obtained in both evaluated countries is the higher prevalence of salt addition in Austrian toddlers. Nearly $90 \%$ of older Austrian toddlers received meals with added salt, and this fact could lead to diminishing gaps in toddlers' characteristics within both subgroups. Moreover, the strength of the factors associated with the use of added salt may also reduce because of the use of added salt increases with toddlers' age. In our previous paper, which was a part of the present study, we also observed that the Austrian mothers were following the WHO recommendations about the age of introduction of complementary feeding less often than the Polish mothers [31].

\subsection{Strengths and Limitations}

An important strength of this study was the large sample size $(n=5893)$ from two European countries. Due to the study design and administration of the questionnaire via the Internet, it was possible to reach out to mothers from diverse parts of Poland and Austria and provide equal access for participation over geographical and financial barriers. Moreover, we assessed the prevalence of the use of added salt, sugar, and both of them in two age categories, which is important because of the fact that children younger than 2 years should not be given added salt nor sugar [10]. Further, our study was able to explore some plausible factors associated with the use of added salt and sugar, both sociodemographic and early feeding, as well as to investigate the association between dietary 
habits among children with different statuses of use of added salt and sugar. It is worth emphasizing that some of the presented factors may be modifiable. This creates the possibility of interventions aimed to improve early dietary habits and even reduce the risk of non-communicable diseases in further life.

Notwithstanding, the present study has some limitations, which should be considered. First of all, the survey was conducted only among Internet users. However, Internet access is generally available in Poland, as well as in Austria. In Poland, $99 \%$ of households with children have Internet access, and in Austria, 88\% [31,74]. Second, all of the data about early feeding factors were self-reported by mothers of toddlers aged 12-36 months and they could have some difficulties in remembering details. Although, existing data suggest that maternal recall is valid years later, whereas information about introducing solids may be poorer reported [75-78]; nonetheless, the recall time in the present study was relatively short ( $\leq 3$ years), therefore, recall bias was minimized. Third, we collected data only about the current use of added salt and sugar. No information about the intake of salt/sodium, free/added sugars, or products that contain high amounts of salt or sugar, as well as the time when salt and sugar were introduced into children's diet is available. Fourth, there is a likelihood that the present investigation involved more mothers who were especially interested in children's nutrition, thereupon they could follow the nutritional recommendations to a higher degree. Nonetheless, the present study demonstrated a low adherence to the recommendation of avoiding salt and sugar during complementary feeding, as we observed a high prevalence of use of added salt (especially in the Austrian toddlers) and moderately high prevalence of use of added sugar. Moreover, we found some interesting influencing factors (e.g., related to early feeding practices or maternal concerns about children eating behaviors), which can be directions to develop intervention strategies and educational programs.

\section{Conclusions}

The most worrisome finding from the present study is that at least half of the Polish and majority of the Austrian mothers, especially of the older toddlers, did not follow the recommendations about avoiding salt use during the first years of children's life. Even though the prevalence of the use of added sugar was lower, those results are also disconcerting, especially as the use of sugar, like salt, should be limited in children's nutrition. Furthermore, the obtained results revealed that the use of added salt, sugar, or both salt and sugar in the toddlers' diet was associated with multiparity and lower maternal age. Besides, we found that shorter duration of exclusive breastfeeding and lack of any or current breastfeeding, as well as traditional spoon-feeding, may increase the risk of use of added salt, sugar, or both of them, whereas introducing solids using the BLW method may decrease it. Especially alarming is that non-compliance with salt and sugar recommendations was related to other unhealthy feeding practices and poorer dietary habits (Western-like vs. pro-health dietary patterns) during infancy and toddlerhood.

As higher salt/sugar intake in childhood potentially leads to becoming accustomed to high levels of saltiness/sweetness in food, this in turn, aids improper food choice later in life. Taking this into account, as well as possible implications for long-term health effects that arise from exceeding salt and sugar consumption, some efforts should be taken to lower intake of those ingredients. Those actions should include creating political, social and food environment that supports the development of healthy dietary habits associated with low intake of added salt and sugar. Our results indicate that those interventions to promote a healthy diet should start very early, ideally at the beginning of introducing complementary foods.

Supplementary Materials: The following are available online at http://www.mdpi.com/1660-4601/17/14/5025/s1, Table S1: Univariate regression analysis models of factors influencing the use of added salt, sugar, and salt and sugar in Polish toddlers $(n=4110)$; Table S2: Univariate regression analysis models of factors influencing the use of added salt, sugar, and salt and sugar in Austrian toddlers $(n=1783)$; Table S3: Sources of knowledge about children nutrition according to the country, age group and use of added salt, sugar, and both salt and sugar. 
Author Contributions: M.A.Z., J.H., and P.R. designed the study; D.M.-K., M.A.Z., P.R., and D.M. was involved in the data acquisition; D.M.-K., and M.A.Z. analyzed and cleaned the data; M.A.Z. and D.M.-K. conducted the statistical analyses; M.A.Z., D.M.-K., and J.H. interpreted the data and wrote the manuscript; J.H., D.M., and P.R. revised the manuscript critically for important intellectual content. All authors have read and agreed to the published version of the manuscript.

Funding: The research was financed by the Polish Ministry of Science and Higher Education within funds of Institute of Human Nutrition Sciences, Warsaw University of Life Sciences (WULS), for scientific research.

Acknowledgments: We thank Jacqueline Bichler for her help during data collection.

Conflicts of Interest: The authors declare that they have no competing interests.

$\begin{array}{ll}\text { Abbreviations } \\ \text { aOR } & \text { Adjusted odds ratio } \\ \text { AS } & \text { Added salt } \\ \text { AS\&Su } & \text { Added salt and sugar } \\ \text { ASu } & \text { Added sugar } \\ \text { BF } & \text { Breastfeeding } \\ \text { BLW } & \text { Baby led weaning } \\ \text { CF } & \text { Complementary foods } \\ \text { CFI } & \text { Complementary feeding introduction } \\ \text { CI } & \text { Confidence interval } \\ \text { DOHaD } & \text { Developmental Origins of Health and Disease hypothesis } \\ \text { EBF } & \text { Exclusive breastfeeding } \\ \text { EFSA } & \text { European Food Safety Authority } \\ \text { GDP } & \text { Gross domestic product } \\ \text { OR } & \text { Odds ratio } \\ \text { TSF } & \text { Traditional spoon-feeding }\end{array}$

\section{References}

1. Baird, J.; Jacob, C.; Barker, M.; Fall, C.H.D.; Hanson, M.; Harvey, N.C.; Inskip, H.M.; Kumaran, K.; Cooper, C. Developmental origins of health and disease: A lifecourse approach to the prevention of non-communicable diseases. Healthcare 2017, 5, 14. [CrossRef] [PubMed]

2. De Cosmi, V.; Scaglioni, S.; Agostoni, C. Early taste experiences and later food choices. Nutrients 2017, 9, 107. [CrossRef] [PubMed]

3. Reidy, K.C.; Deming, D.M.; Briefel, R.R.; Fox, M.K.; Saavedra, J.M.; Eldridge, A.L. Early development of dietary patterns: Transitions in the contribution of food groups to total energy-Feeding Infants and Toddlers Study, 2008. BMC Nutr. 2017, 3, 5. [CrossRef]

4. Daniels, L.A. Feeding practices and parenting: A pathway to child health and family happiness. Ann. Nutr. Metab. 2019, 74, 29-42. [CrossRef]

5. Venditti, C.; Musa-Veloso, K.; Lee, H.Y.; Poon, T.; Mak, A.; Darch, M.; Juana, J.; Fronda, D.; Noori, D.; Pateman, E.; et al. Determinants of sweetness preference: A scoping review of human studies. Nutrients 2020, 12, 718. [CrossRef]

6. Liem, D.G. Infants' and children's salt taste perception and liking: A review. Nutrients 2017, 9, 1011. [CrossRef]

7. Gidding, S.S.; Dennison, B.A.; Birch, L.L.; Daniels, S.R.; Gilman, M.W.; Lichtenstein, A.H.; Rattay, K.T.; Steinberger, J.; Stettler, N.; Van Horn, L. Dietary recommendations for children and adolescents: A guide for practitioners: Consensus statement from the American Heart Association. Circulation 2005, 112, 2061-2075. [CrossRef]

8. Fewtrell, M.; Bronsky, J.; Campoy, C.; Domellöf, M.; Embleton, N.; Mis, N.F.; Hojsak, I.; Hulst, J.M.; Indrio, F.; Lapillonne, A.; et al. Complementary feeding: A position paper by the European Society for Paediatric Gastroenterology, Hepatology, and Nutrition (ESPGHAN) committee on nutrition. J. Pediatr. Gastroenterol. Nutr. 2017, 64, 119-132. [CrossRef] 
9. Fidler Mis, N.; Braegger, C.; Bronsky, J.; Campoy, C.; Domellöf, M.; Embleton, N.D.; Hojsak, I.; Hulst, J.; Indrio, F.; Lapillonne, A.; et al. Sugar in infants, children and adolescents: A position paper of the European Society for Paediatric Gastroenterology, Hepatology and Nutrition Committee on Nutrition. J. Pediatr. Gastroenterol. Nutr. 2017, 65, 681-696. [CrossRef]

10. WHO. Commercial Foods for Infants and Young Children in the WHO European Region. A Study of the Availability, Composition and Marketing of Baby Foods in Four European Countries; World Health Organization: Geneva, Switzerland, 2019.

11. Leyvraz, M.; Chatelan, A.; da Costa, B.R.; Taffé, P.; Paradis, G.; Bovet, P.; Bochud, M.; Chiolero, A. Sodium intake and blood pressure in children and adolescents: A systematic review and meta-analysis of experimental and observational studies. Int. J. Epidemiol. 2018, 47, 1796-1810. [CrossRef]

12. Vos, M.B.; Kaar, J.L.; Welsh, J.A.; Van Horn, L.V.; Feig, D.I.; Anderson, C.A.M.; Patel, M.J.; Cruz Munos, J.; Krebs, N.F.; Xanthakos, S.A.; et al. Added sugars and cardiovascular disease risk in children: A scientific statement from the American Heart Association. Circulation 2017, 135, e1017-e1034. [CrossRef] [PubMed]

13. Moosavian, S.P.; Haghighatdoost, F.; Surkan, P.J.; Azadbakht, L. Salt and obesity: A systematic review and meta-analysis of observational studies. Int. J. Food Sci. Nutr. 2017, 68, 265-277. [CrossRef] [PubMed]

14. Poorolajal, J.; Sahraei, F.; Mohamdadi, Y.; Doosti-Irani, A.; Moradi, L. Behavioral factors influencing childhood obesity: A systematic review and meta-analysis. Obes. Res. Clin. Pract. 2020, 14, 109-118. [CrossRef] [PubMed]

15. Peres, M.A.; Sheiham, A.; Liu, P.; Demarco, F.F.; Silva, A.E.R.; Assunção, M.C.; Menezes, A.M.; Barros, F.C.; Peres, K.G. Sugar consumption and changes in dental caries from childhood to adolescence. J. Dent. Res. 2016, 95, 388-394. [CrossRef] [PubMed]

16. Noble, E.E.; Hsu, T.M.; Jones, R.B.; Fodor, A.A.; Goran, M.I.; Kanoski, S.E. Early-life sugar consumption affects the rat microbiome independently of obesity. J. Nutr. 2017, 147, 20-28. [CrossRef]

17. Rinninella, E.; Cintoni, M.; Raoul, P.; Lopetuso, L.R.; Scaldaferri, F.; Pulcini, G.; Miggiano, G.A.D.; Gasbarrini, A.; Mele, M.C. Food components and dietary habits: Keys for a healthy gut microbiota composition. Nutrients 2019, 11, 2393. [CrossRef]

18. Stiemsma, L.T.; Michels, K.B. The Role of the microbiome in the developmental origins of health and disease. Pediatrics 2018, 141. [CrossRef]

19. Nehring, I.; Kostka, T.; von Kries, R.; Rehfuess, E.A. Impacts of in utero and early infant taste experiences on later taste acceptance: A systematic review. J. Nutr. 2015, 145, 1271-1279. [CrossRef]

20. Stein, L.J.; Cowart, B.J.; Beauchamp, G.K. The development of salty taste acceptance is related to dietary experience in human infants: A prospective study. Am. J. Clin. Nutr. 2012, 94, 123-129. [CrossRef]

21. Brown, I.J.; Tzoulaki, I.; Candeias, V.; Elliott, P. Salt intakes around the world: Implications for public health. Int. J. Epidemiol. 2009, 38, 791-813. [CrossRef]

22. Foterek, K.; Buyken, A.E.; Bolzenius, K.; Hilbig, A.; Nöthlings, U.; Alexy, U. Commercial complementary food consumption is prospectively associated with added sugar intake in childhood. Br. J. Nutr. 2016, 115, 2067-2074. [CrossRef]

23. Kyttälä, P.; Erkkola, M.; Kronberg-Kippilä, C.; Tapanainen, H.; Veijola, R.; Simell, O.; Knip, M.; Virtanen, S.M. Food consumption and nutrient intake in Finnish 1-6-year-old children. Public Health Nutr. 2010, 13, 947-956. [CrossRef] [PubMed]

24. Grimes, C.A.; Campbell, K.J.; Riddell, L.J.; Nowson, C.A. Sources of sodium in Australian children's diets and the effect of the application of sodium targets to food products to reduce sodium intake. Br. J. Nutr. 2011, 105, 468-477. [CrossRef] [PubMed]

25. Campbell, K.J.; Hendrie, G.; Nowson, C.; Grimes, C.A.; Riley, M.; Lioret, S.; McNaughton, S.A. Sources and correlates of sodium consumption in the first 2 years of life. J. Acad. Nutr. Diet. 2014, 114, 1525-1532. [CrossRef]

26. Cribb, V.L.; Warren, J.M.; Emmett, P.M. Contribution of inappropriate complementary foods to the salt intake of 8-month-old infants. Eur. J. Clin. Nutr. 2012, 66, 104-110. [CrossRef]

27. Bournez, M.; Ksiazek, E.; Charles, M.A.; Lioret, S.; Brindisi, M.C.; De Lauzon-Guillain, B.; Nicklaus, S. Frequency of use of added sugar, salt, and fat in infant foods up to 10 months in the nationwide ELFE cohort study: Associated infant feeding and caregiving practices. Nutrients 2019, 11, 733. [CrossRef] [PubMed] 
28. Verduci, E.; Banderali, G.; Montanari, C.; Canani, R.B.; Caserta, L.C.; Corsello, G.; Mosca, F.; Piazzolla, R.; Rescigno, M.; Terracciano, L.; et al. Childhood dietary intake in Italy: The epidemiological “MY FOOD DIARY" survey. Nutrients 2019, 11, 1129. [CrossRef] [PubMed]

29. Pietrobelli, A.; Agosti, M.; Palmer, C.; Pereira-Da-Silva, L.; Rego, C.; Rolland-Cacherà, M.F.; Zuccotti, G. Nutrition in the first 1000 days: Ten practices to minimize obesity emerging from published science. Int. J. Environ. Res. Public Health 2017, 14, 1491. [CrossRef]

30. Song, P.; Zhang, Y.; Yu, J.; Zha, M.; Zhu, Y.; Rahimi, K.; Rudan, I. Global prevalence of hypertension in children: A systematic review and meta-analysis. JAMA Pediatr. 2019, 173, 1154-1163. [CrossRef]

31. Zielinska, M.A.; Rust, P.; Masztalerz-Kozubek, D.; Bichler, J.; Hamulka, J. Factors Influencing the Age of Complementary Feeding-A Cross-Sectional Study from Two European Countries. Int. J. Environ. Res. Public Health 2019, 16, 3799. [CrossRef]

32. Eurostat. GDP at Regional Level. Available online: https://ec.europa.eu/eurostat/statistics-explained/index. php/GDP_at_regional_level\#Regional_GDP_per_capita (accessed on 27 May 2020).

33. WHO. The World Health Organization's Infant Feeding Recommendation. Available online: https://www. who.int/nutrition/topics/infantfeeding_recommendation/en/ (accessed on 26 May 2020).

34. Fein, S.B.; Labiner-Wolfe, J.; Scanlon, K.S.; Grummer-Strawn, L.M. Selected complementary feeding practices and their association with maternal education. Pediatrics 2008, 122, S91-S97. [CrossRef]

35. Bandara, T.; Hettiarachchi, M.; Liyanage, C.; Amarasena, S. Current infant feeding practices and impact on growth in babies during the second half of infancy. J. Hum. Nutr. Diet. 2015, 28, 366-374. [CrossRef]

36. EFSA. Dietary Salt/Sodium. Available online: https://ec.europa.eu/jrc/en/health-knowledge-gateway/ promotion-prevention/nutrition/salt (accessed on 27 May 2020).

37. Herrick, K.A.; Fryar, C.D.; Hamner, H.C.; Park, S.; Ogden, C.L. Added sugars intake among US infants and toddlers. J. Acad. Nutr. Diet. 2019, 120, 23-32. [CrossRef]

38. Erickson, L.W.; Taylor, R.W.; Haszard, J.J.; Fleming, E.A.; Daniels, L.; Morison, B.J.; Leong, C.; Fangupo, L.J.; Wheeler, B.J.; Taylor, B.J.; et al. Impact of a modified version of baby-led weaning on infant food and nutrient intakes: The BLISS randomized controlled trial. Nutrients 2018, 10, 740. [CrossRef]

39. Wang, Y.; Guglielmo, D.; Welsh, J.A. Consumption of sugars, saturated fat, and sodium among US children from infancy through preschool age, NHANES 2009-2014. Am. J. Clin. Nutr. 2018, 108, 868-877. [CrossRef]

40. Marinho, A.R.; Severo, M.; Correia, D.; Lobato, L.; Vilela, S.; Oliveira, A.; Ramos, E.; Torres, D.; Lopes, C. Total, added and free sugar intakes, dietary sources and determinants of consumption in Portugal: The National Food, Nutrition and Physical Activity Survey (IAN-AF 2015-2016). Public Health Nutr. 2019, 23, 869-881. [CrossRef]

41. Perrar, I.; Schmitting, S.; Della Corte, K.W.; Buyken, A.E.; Alexy, U. Age and time trends in sugar intake among children and adolescents: Results from the DONALD study. Eur. J. Nutr. 2019, 59, 1043-1054. [CrossRef]

42. Brion, M.J.; Ness, A.R.; Davey Smith, G.; Emmett, P.; Rogers, I.; Whincup, P.; Lawlor, D.A. Sodium intake in infancy and blood pressure at 7 years: Findings from the Avon longitudinal study of parents and children. Eur. J. Clin. Nutr. 2008, 62, 1162-1169. [CrossRef]

43. Weker, H.; Brudnicka, E.; Barańska, M.; Rowicka, G.; Strucińska, M.; Więch, M.; Dylag, H.; Klemarczyk, W.; Socha, P.; Mazur, J. Dietary patterns of children aged 1-3 years in Poland in two population studies. Ann. Nutr. Metab. 2019, 75, 66-76. [CrossRef] [PubMed]

44. Bailey, R.L.; Fulgoni, V.L.; Cowan, A.E.; Gaine, P.C. Sources of added sugars in young children, adolescents, and adults with low and high intakes of added sugars. Nutrients 2018, 10, 102. [CrossRef]

45. Amoutzopoulos, B.; Steer, T.; Roberts, C.; Collins, D.; Page, P. Free and added sugar consumption and adherence to guidelines: The UK national diet and nutrition survey (2014/15-2015/16). Nutrients 2020, 12, 393. [CrossRef]

46. Quader, Z.S.; Gillespie, C.; Sliwa, S.A.; Ahuja, J.K.C.; Burdg, J.P.; Moshfegh, A.; Pehrsson, P.R.; Gunn, J.P.; Mugavero, K.; Cogswell, M.E. Sodium Intake among US School-Aged Children: National Health and Nutrition Examination Survey, 2011-2012. J. Acad. Nutr. Diet. 2017, 176, 139-148. [CrossRef]

47. Yuan, W.L.; Nicklaus, S.; Lioret, S.; Lange, C.; Forhan, A.; Heude, B.; Charles, M.A.; De Lauzon-Guillain, B. Early factors related to carbohydrate and fat intake at 8 and 12 months: Results from the EDEN mother-child cohort. Eur. J. Clin. Nutr. 2017, 71, 219-226. [CrossRef] [PubMed] 
48. Morinaga, Y.; Tsuchihashi, T.; Ohta, Y.; Matsumura, K. Salt intake in 3-year-old Japanese children. Hypertens. Res. 2011, 34, 836-839. [CrossRef]

49. Synnott, K.; Bogue, J.; Edwards, C.A.; Scott, J.A.; Higgins, S.; Norin, E.; Frias, D.; Amarri, S.; Adam, R. Parental perceptions of feeding practices in five European countries: An exploratory study. Eur. J. Clin. Nutr. 2007, 61, 946-956. [CrossRef] [PubMed]

50. Tromp, I.I.M.; Briedé, S.; Kiefte-de Jong, J.C.; Renders, C.M.; Jaddoe, V.W.V.; Franco, O.H.; Hofman, A.; Raat, H.; Moll, H.A. Factors associated with the timing of introduction of complementary feeding: The Generation R Study. Eur. J. Clin. Nutr. 2013, 67, 625-630. [CrossRef]

51. Klingberg, S.; Ludvigsson, J.; Brekke, H.K. Introduction of complementary foods in Sweden and impact of maternal education on feeding practices. Public Health Nutr. 2017, 20, 1054-1062. [CrossRef] [PubMed]

52. Gerritsen, S.; Wall, C.R.; Grant, C.C.; Teixeira, J.A.; Marchioni, D.M.; Pillai, A.; Morton, S.M.B.; de Castro, T.G. Inequalities in infant feeding practices among the growing up in New Zealand cohort. Proceedings 2019, 8, 54. [CrossRef]

53. Maciel, B.L.L.; Moraes, M.L.; Soares, A.M.; Cruz, I.F.S.; De Andrade, M.I.R.; Filho, J.Q.; Junior, F.S.; Costa, P.N.; Abreu, C.B.; Ambikapathi, R.; et al. Infant feeding practices and determinant variables for early complementary feeding in the first 8 months of life: Results from the Brazilian MAL-ED cohort site. Public Health Nutr. 2018, 21, 2462-2470. [CrossRef]

54. Armstrong, J.; Abraham, E.C.; Squair, M.; Brogan, Y.; Merewood, A. Exclusive breastfeeding, complementary feeding, and food choices in UK infants. J. Hum. Lact. 2014, 30, 201-208. [CrossRef]

55. de Almeida Fonseca, P.C.; Ribeiro, S.A.V.; Andreoli, C.S.; de Carvalho, C.A.; Pessoa, M.C.; de Novaes, J.F.; Priore, S.E.; Franceschini, S.D.C.C. Association of exclusive breastfeeding duration with consumption of ultra-processed foods, fruit and vegetables in Brazilian children. Eur. J. Nutr. 2019, 58, 2887-2894. [CrossRef]

56. Kiefte-De Jong, J.C.; De Vries, J.H.; Bleeker, S.E.; Jaddoe, V.W.V.; Hofman, A.; Raat, H.; Moll, H.A. Socio-demographic and lifestyle determinants of "Western-like" and "Health conscious" dietary patterns in toddlers. Br. J. Nutr. 2013, 109, 137-147. [CrossRef]

57. Fegan, S.; Bassett, E.; Peng, Y.; Steel O'Connor, K. Adherence to complementary feeding recommendations for infants and implications for public health. Public Health Nutr. 2016, 19, 638-649. [CrossRef]

58. Elliott, C.D.; Conlon, M.J. Packaged baby and toddler foods: Questions of sugar and sodium. Pediatr. Obes. 2015, 10, 149-155. [CrossRef] [PubMed]

59. Maalouf, J.; Cogswell, M.E.; Bates, M.; Yuan, K.; Scanlon, K.S.; Pehrsson, P.; Gunn, J.P.; Merritt, R.K. Sodium, sugar, and fat content of complementary infant and toddler foods sold in the United States, 2015. Am. J. Clin. Nutr. 2017, 105, 1443-1452. [CrossRef] [PubMed]

60. Hollis, J.L.; Crozier, S.R.; Inskip, H.M.; Cooper, C.; Godfrey, K.M.; Robinson, S.M. Age at introduction of solid foods and feeding difficulties in childhood: Findings from the Southampton Women's Survey. Br. J. Nutr. 2016, 116, 743-750. [CrossRef]

61. Morison, B.J.; Heath, A.L.M.; Haszard, J.J.; Hein, K.; Fleming, E.A.; Daniels, L.; Erickson, E.W.; Fangupo, L.J.; Wheeler, B.J.; Taylor, B.J.; et al. Impact of a modified version of baby-led weaning on dietary variety and food preferences in infants. Nutrients 2018, 10, 1092. [CrossRef]

62. Morison, B.J.; Taylor, R.W.; Haszard, J.J.; Schramm, C.J.; Erickson, L.W.; Fangupo, L.J.; Fleming, E.A.; Luciano, A.; Heath, A.L.M. How different are baby-led weaning and conventional complementary feeding? A cross-sectional study of infants aged 6-8 months. BMJ Open 2016, 6. [CrossRef]

63. Rowan, H.; Lee, M.; Brown, A. Differences in dietary composition between infants introduced to complementary foods using Baby-led weaning and traditional spoon feeding. J. Hum. Nutr. Diet. 2019, 32, 11-20. [CrossRef]

64. Brown, A. Differences in eating behaviour, well-being and personality between mothers following baby-led vs. traditional weaning styles. Matern. Child Nutr. 2016, 12, 826-837. [CrossRef]

65. Fu, X.; Conlon, C.A.; Haszard, J.J.; Beck, K.L.; von Hurst, P.R.; Taylor, R.W.; Heath, A.L.M. Food fussiness and early feeding characteristics of infants following Baby-Led Weaning and traditional spoon-feeding in New Zealand: An internet survey. Appetite 2018, 130, 110-116. [CrossRef]

66. Komninou, S.; Halford, J.C.G.; Harrold, J.A. Differences in parental feeding styles and practices and toddler eating behaviour across complementary feeding methods: Managing expectations through consideration of effect size. Appetite 2019, 137, 198-206. [CrossRef] [PubMed] 
67. Grimes, C.A.; Riddell, L.J.; Campbell, K.J.; Nowson, C.A. Dietary salt intake, sugar-sweetened beverage consumption, and obesity risk. Pediatrics 2013, 131, 14-21. [CrossRef]

68. Marrero, N.M.; He, F.J.; Whincup, P.; MacGregor, G.A. Salt intake of children and adolescents in south london consumption levels and dietary sources. Hypertension 2014, 63, 1026-1032. [CrossRef] [PubMed]

69. Rios-Leyvraz, M.; Bovet, P.; Bochud, M.; Genin, B.; Russo, M.; Rossier, M.F.; Tabin, R.; Chiolero, A. Estimation of salt intake and excretion in children in one region of Switzerland: A cross-sectional study. Eur. J. Nutr. 2019, 58, 2921-2928. [CrossRef] [PubMed]

70. Mallan, K.M.; Fildes, A.; Magarey, A.M.; Daniels, L.A. The relationship between number of fruits, vegetables, and noncore foods tried at age 14 months and food preferences, dietary intake patterns, fussy eating behavior, and weight status at age 3.7 years. J. Acad. Nutr. Diet. 2016, 116, 630-637. [CrossRef] [PubMed]

71. Khokhar, D.; Nowson, C.A.; Margerison, C.; Bolam, B.; Grimes, C.A. Knowledge and attitudes are related to selected salt-specific behaviours among Australian parents. Nutrients 2018, 10, 720. [CrossRef] [PubMed]

72. Mennella, J.A.; Finkbeiner, S.; Lipchock, S.V.; Hwang, L.-D.; Reed, D.R. Preferences for salty and sweet tastes are elevated and related to each other during childhood. PLoS ONE 2014, 9, e92201. [CrossRef] [PubMed]

73. Bouhlal, S.; Issanchou, S.; Nicklaus, S. The impact of salt, fat and sugar levels on toddler food intake. Br. J. Nutr. 2011, 105, 645-653. [CrossRef]

74. Eurostat Individuals-Internet Use. Available online: https://appsso.eurostat.ec.europa.eu/nui/show.do? dataset=isoc_ci_ifp_iu\&lang=en (accessed on 25 May 2020).

75. Li, R.; Scanlon, K.S.; Serdula, M.K. The validity and reliability of maternal recall of breastfeeding practice. Nutr. Rev. 2005, 63, 103-110. [CrossRef]

76. Herrmann, D.; Suling, M.; Reisch, L.; Siani, A.; De Bourdeaudhuij, I.; Maes, L.; Santaliestra-Pasías, A.M.; Veidebaum, T.; Molnár, D.; Pala, V.; et al. Repeatability of maternal report on prenatal, perinatal and early postnatal factors: Findings from the IDEFICS parental questionnaire. Int. J. Obes. 2011, 35, 52-60. [CrossRef] [PubMed]

77. Natland, S.T.; Andersen, L.F.; Nilsen, T.I.L.; Forsmo, S.; Jacobsen, G.W. Maternal recall of breastfeeding duration twenty years after delivery. BMC Med. Res. Methodol. 2012, 12, 179. [CrossRef] [PubMed]

78. Van Zyl, Z.; Maslin, K.; Dean, T.; Blaauw, R.; Venter, C. The accuracy of dietary recall of infant feeding and food allergen data. J. Hum. Nutr. Diet. 2016, 29, 777-785. [CrossRef] [PubMed]

(C) 2020 by the authors. Licensee MDPI, Basel, Switzerland. This article is an open access article distributed under the terms and conditions of the Creative Commons Attribution (CC BY) license (http://creativecommons.org/licenses/by/4.0/). 